\title{
On Damage Identification in Planar Frames of Arbitrary Size
}

\author{
I. Fiore $\left(\mathbb{D},{ }^{1}\right.$ A. Greco $\mathbb{D}^{1}{ }^{1}$ and A. Pluchino $\mathbb{D}^{2,3}$ \\ ${ }^{1}$ Department of Civil Engineering and Architecture, University of Catania, Via S. Sofia 64, Catania, Italy \\ ${ }^{2}$ Department of Physics and Astronomy "Ettore Majorana", University of Catania, Via S. Sofia 64, Catania, Italy \\ ${ }^{3}$ INFN, Unit of Catania, Catania, Italy \\ Correspondence should be addressed to I. Fiore; ilaria.fiore@phd.unict.it
}

Received 1 September 2021; Revised 21 December 2021; Accepted 22 December 2021; Published 20 January 2022

Academic Editor: C. M. Wang

Copyright (c) 2022 I. Fiore et al. This is an open access article distributed under the Creative Commons Attribution License, which permits unrestricted use, distribution, and reproduction in any medium, provided the original work is properly cited.

\begin{abstract}
Framed structures are deeply studied in civil engineering since they provide a numerical model for the analysis of the static and dynamic response of multi-storey buildings. In order to evaluate the vibrational properties of these structures, an eigen-problem, which involves the stiffness and mass matrices of the frame, must be solved. Both matrices can be assembled by means of standard methods, which take into account the numbers of degrees of freedom of the frame. The occurrence of concentrated damage in some vulnerable sections modifies the degrees of freedom and therefore both the stiffness and mass matrices. Very often, the critical sections are located in the joints between the structural elements of the frame where the bending moment reaches its maximum value. Assuming that the joints are rigid in the undamaged configuration of the frame, it is possible to take into account their loss of stiffness due to the presence of eventual damage by means of hinges with rotational springs of variable rigidity. In this paper, an original algorithm that allows us to evaluate the stiffness and mass matrices and therefore the natural frequencies of vibration of undamaged and damaged planar frames with an arbitrary number of beams and columns is presented. The proposed algorithm for the stiffness and mass matrices determination requires a few input data which can be provided in a text file and therefore allows us to speed up the procedure with respect to the application of an FEM approach which requires the construction of single models for each considered frame. The results obtained by means of the proposed algorithm have been validated through a comparison with those provided by an FEM model implemented in SAP2000. The natural frequencies obtained by means of the proposed approach are used for the solution of two different inverse problems, which concern the identification of, respectively, the mechanical characteristics of the constitutive material and the location and intensity of the damage. Both the proposed identification procedures deal with optimization algorithms that are based on opportune fitness functions. Applications to frames of different size confirm the validity of the presented identification algorithms. Furthermore, an iterative procedure, able to reduce the required computational burden related to the identification of the location and intensity of damage, is presented and applied in a parametric study concerning frames with increasing size.
\end{abstract}

\section{Introduction}

Due to the aging of a very high number of existing structures in the urbanized areas of the various continents, it has been necessary over the years to verify their conditions of integrity. The repeated stresses to which the structures are subjected and the action of atmospheric agents can cause damage of varying intensity, which reduces the bearing capacity of structures and inevitably leads to structural failure. The object of study, usually called "structural health monitoring," has therefore attracted a lot of interest from researchers who have published numerous scientific papers.
The key point in all the procedures concerning damaged structures consists in the definition of an accurate and reliable numerical model of the structure.

A great number of studies are based on the comparison of the static or dynamic response of models of damaged structures with respect to the undamaged configuration [1-9] and propose damage identification techniques. The latter often require the measurement of different kind of data on the existing structure; these, for example, may concern the variation of dynamic characteristics, such as natural frequencies [10-20], mode shapes [21, 22], modal curvature $[23,24]$, and time-frequency features [25], or static quantities, such as displacements or strains induced by applied 
loads [26-28]. By means of the interpretation of the measured responses, these approaches allow us to identify the presence of structural damage without the need to perform visual inspections or destructive investigations.

Damage identification procedures are often based on the solution of an inverse problem that compares numerical response data, evaluated on a model of the structure, with the corresponding experimentally measured ones [29-31]. The interest of the scientific community in damage identification is highlighted by the great variety of presented computational procedures such as genetic algorithms [32-34], metaheuristic algorithms [35], particle swarm optimization [36], fuzzy cognitive maps [37], digital image correlation [38], vision-based measurements [39], neural networks [40], and wavelet analysis [41].

In order to build a realistic numerical model of the damaged structure, it is necessary to accurately model the damage itself; numerous attempts to characterize and quantify local defects are reported in the literature. All the models involve a reduction of the stiffness of the structural element by using one-dimensional continuum theories [42] or fracture mechanics methods $[43,44]$. The damage can be either localized or diffused on a certain portion of the length of the structural element, and its intensity can be related to the reduction of the reactive area of the cross section, for example, due to the presence of a notch. Some contributions on crack modeling approaches and their effects on the response of beams can be found in $[45,46]$. Several papers consider the joints between beams and columns as possible damage locations [33, 47-49].

The aim of the present study is to give a contribution to the evaluation of reliable numerical models of undamaged and damaged frames with arbitrary numbers of beams and columns. In particular, the stiffness and mass matrices of undamaged and damaged frames of variable size are here evaluated by means of some original algorithms. These algorithms allow us to analyse frames with arbitrary numbers of columns and floors, only providing a text file in which geometrical and mechanical characteristics of the structural elements are reported. It is interesting to highlight that this method allows us to significantly speed up the procedure with respect to standard FEM approaches which require the construction of single models for each considered frame.

Considering that early detection of the damage location is very important in order to prevent its progressive expansion, single damage is here considered. This damage is assumed to be concentrated in a transversal section of one of the structural elements, and the related stiffness reduction is modeled by means of a hinge with a rotational spring. The stiffness of the spring decreases with the intensity of the damage. This damage model is often adopted in the scientific literature since it provides reliable results validated also by means of experimental tests [12, 48, 50, 51]. A discrete number of critical sections located in the ends of columns or beams have been taken into account as possible damage location. This assumption is based on the consideration that the bending moment usually reaches its maximum value in these cross sections, particularly if the frame has been subjected to increasing variable forces due to seismic excitations $[52,53]$. The stiffness and mass matrices of undamaged and damaged frames (in which one hinge with rotational spring is located in an arbitrary critical section) have been evaluated by means of the proposed algorithms and used to determine the natural frequencies of vibration of undamaged and damaged frames. The results in terms of static displacements and natural frequencies of undamaged and damaged frames have been validated by means of a comparison with the correspondent results obtained adopting an FEM model implemented with the software SAP2000.

Two optimization problems based on the evaluation of natural frequencies of frames with arbitrary size have been proposed. In particular, the mechanical parameters of the constitutive material have been calibrated by means of the solution of an optimization problem that takes into account the natural frequencies of the undamaged frame.

Furthermore, a computational method for detecting the first damage in planar frames of arbitrary size is also proposed according to the procedure presented in [54]. This approach is based on the assumption that appropriate sensors are permanently located on the frame and periodically measure its vibrational properties. Knowing the current natural frequencies, it will be possible to immediately detect the appearance of the first structural damage when a difference between these frequencies and those numerically evaluated for the undamaged frame is observed. In particular, assigning to each critical section an opportune fitness function, the optimization procedure seeks for the absolute maximum value. Examples of some case studies represented by different frames together with a parametric study, in which the size of the frame progressively increases of one floor and one span, are presented. In the latter study, the damage has been virtually located either at the base of a fixed column or at the end of a beam, and it has been shown that the related fitness functions always reach their maximum value for the correct damage location and intensity. Furthermore, for frames of large size having a great number of critical sections, an automatic procedure, which allows us to iteratively converge to the exact damage position without evaluating all the fitness functions, and therefore reducing the computational burden, is illustrated.

\section{Undamaged Frames of Arbitrary Size}

In the present study, planar regular frames, with columns clamped at the ground level, are considered. These are characterized by arbitrary numbers $N_{f}$ and $N_{c}$, respectively, for floors and columns.

In the following, it will be assumed that all the columns in the $i$-th interstorey have height $H_{i}$, while the length $L_{j}$ of the beams may vary in the interstorey (Figure 1).

Furthermore, the moments of inertia of the cross sections of beams and columns will be denoted, respectively, by $I_{b i}$ and $I_{c i}$. Distributed masses $m_{b i}$ and $m_{c i}$, respectively, for beam and column at the $i$-th floor are taken into account.

Neglecting the axial deformability of the structural members, the degrees of freedom of the frame correspond to 


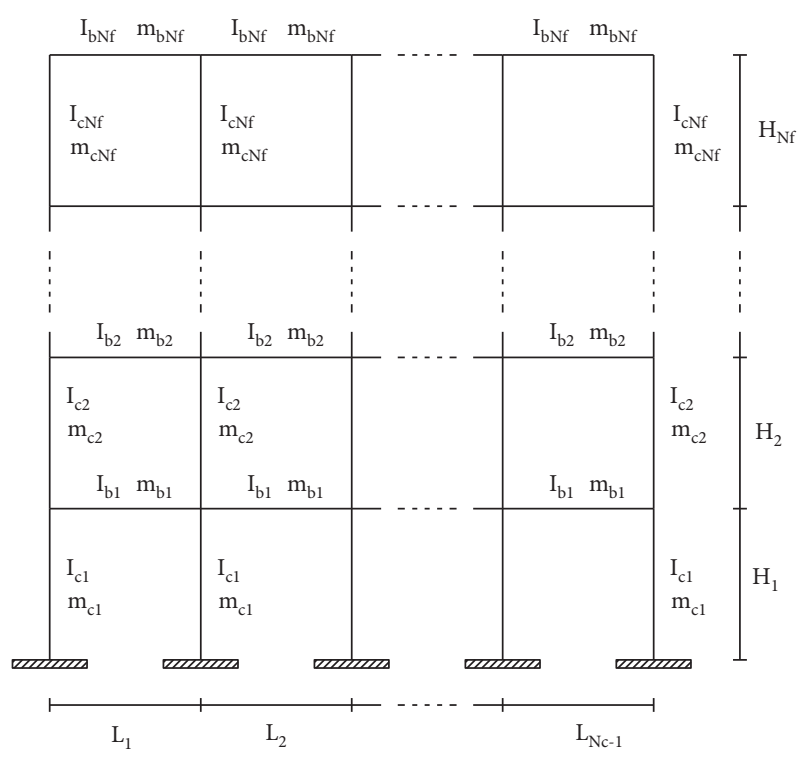

FIgURE 1: Frame geometry.

the horizontal displacements of each floor and the rotation of the unconstrained nodes. The number of degrees of freedom of the undamaged frame and the size of the stiffness and mass matrices is as follows:

$$
S_{u}=N_{f}\left(1+N_{c}\right) \text {. }
$$

Each component of the stiffness matrix $\mathbf{K}^{u}$ and mass matrix $\mathbf{M}^{u}$ is determined by evaluating the nodal forces associated with each degree of freedom. These matrices are reported in Appendix A and B for the sake of clarity.

Once the stiffness and mass matrices for the undamaged frame are evaluated, the $S_{u}$ natural frequencies of vibration $\omega_{u}$ and the corresponding modes $\varphi_{u}$ of the undamaged frame can be evaluated, solving the classic equation:

$$
\left(\mathbf{K}^{u}-\omega_{u}^{2} \mathbf{M}^{u}\right) \boldsymbol{\varphi}_{u}=0
$$

2.1. Identification of Material Parameters. Assuming that the constitutive material of the analysed frame has a linear elastic behaviour, two characteristic parameters, such as Young's modulus $E$ and the specific weight $\mu$, can be evaluated by means of an optimization procedure. In particular, an opportune objective function must be minimized. This function measures the difference between the first three calculated frequencies of vibration $\omega_{c}$ (numerically evaluated varying $E$ and $\mu$ around some characteristic average values) and the experimentally measured ones $\omega_{e}$.

$$
O(E, \mu)=\sum_{i=1}^{3}\left(\frac{\omega_{c i}(E, \mu)-\omega_{e i}}{\omega_{e i}}\right)^{2} .
$$

It is worth noting that, for a different constitutive material, other characteristic parameters can be evaluated with a similar procedure.

\section{Damaged Frames of Arbitrary Size}

In this study, reference is made to single damage, which can be located in one of the critical sections of the frame corresponding with the base or the top of each column at each inter-storey or the ends of each beam. With reference to the two corners at the top of the frame, the critical section will be located in correspondence with the structural member (beam or column) having the lower elastic moment.

The total number of critical sections in which the damage could be located is as follows:

$$
N_{d}=2 N_{c} \cdot N_{f}+2 N_{f}\left(N_{c}-1\right)-2 .
$$

In accordance with what is frequently adopted in the scientific community, the loss of resistance induced by the presence of the damage is modeled by means of a hinge with a rotational spring whose stiffness $k_{\varphi}$ is related to the intensity of the damage $[12,48,50,51]$.

In order to evaluate the natural frequencies of the frame with an arbitrary number of beams and columns having damage located in one of the previously described critical sections, new stiffness and mass matrices must be assembled as described in the following.

Figure 2 shows an example represented by a two-storey frame and reports, from left to right, its 8 degrees of freedom in the undamaged configuration, the 18 critical sections, and the 9 degrees of freedom in the damaged configuration corresponding, for example, to damage located in the critical section labeled as 5 .

For each location of the damage, stiffness and mass matrices, respectively, denoted as $\mathbf{K}^{d}$ and $\mathbf{M}^{d}$, are evaluated. These matrices are assembled analogously to what is described for the undamaged frame with some differences in the components that involve the moment in the rotational spring, as described in Appendix C.

It is worth pointing out that the insertion of a hinge with a rotational spring to model the damage will increase the number of degrees of freedom, and therefore, the size of the stiffness and mass matrices by one unit with respect to the undamaged frame $\left(S_{d}=S_{u}+1\right)$.

Therefore, the following $S_{d}$ equations must be solved in order to calculate the frequencies and modes of vibration for all the possible configurations of the damaged frame.

$$
\left(\mathbf{K}^{d}-\omega_{d}^{2} \mathbf{M}^{d}\right) \boldsymbol{\varphi}_{d}=0
$$

The stiffness of the rotational spring modeling the damage will be assumed to be equal to $n_{i}$ discrete values, which decrease as the intensity of the damage increases.

In particular, the stiffness of the rotational spring is expressed as $[48,51]$

$$
k_{\varphi i}=\alpha_{i} \frac{E I_{d}}{L_{d}}, \quad i=1, \ldots, n_{i},
$$

where $\alpha_{i}$ is an integer number and $I_{d}$ and $L_{d}$ are, respectively, the moment of inertia and the length of the structural element where the damage is located (beam or column). 


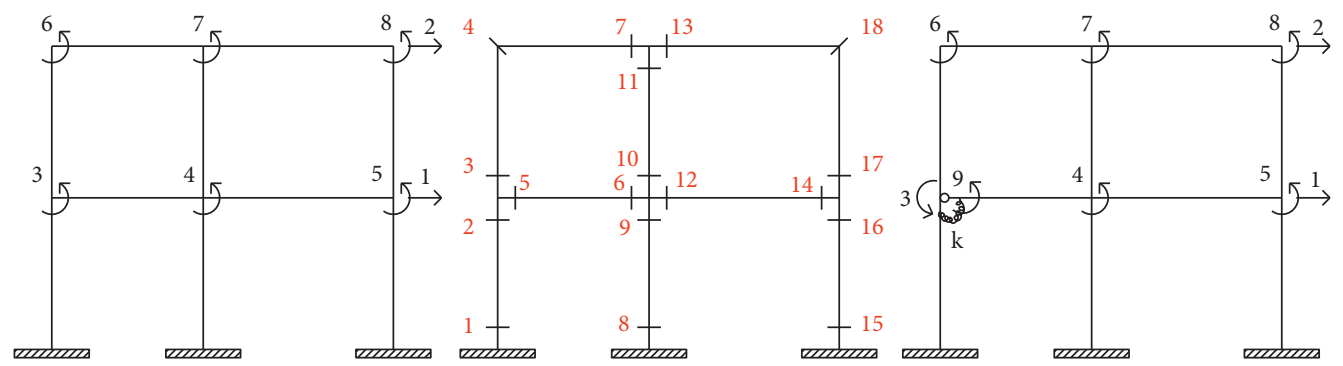

FIgURE 2: Degrees of freedom and critical sections of a frame with one damage.

\section{Validation of the Proposed Approach}

In order to validate the results obtained by means of the proposed routines for the construction of both stiffness and mass matrices for damaged and undamaged frames, some numerical applications are presented here. In particular, a comparison of static displacements and frequencies of vibration obtained through the proposed matrices and by means of an FEM discretization of the frame with SAP2000 [55] has been performed, showing a good correspondence.

The five-storey steel frame reported in Figure 3 has been considered for this validation. The degrees of freedom for the considered symmetric frame in its undamaged configuration are twenty as shown in Figure 4(a). Commercial steel profiles are considered for beams and columns and indicated in Figure 3, which also reports the length of the structural members. In Table 1, moments of inertia and the ratio between elastic moments of the cross sections and the yield stress are reported together with the values of the distributed masses.

An example of the damaged configuration of the frame is reported in Figure 4(b). Damage located at the base of the left column of the first floor has been assumed. The intensity of the damage has been represented by the stiffness intensity parameter $\alpha=25$.

The static response has been determined considering a distributed load equal to $50 \mathrm{kN} / \mathrm{m}$ acting on the beams and a concentrated force equal to $400 \mathrm{kN}$ acting at each floor level.

The static response in terms of floor displacements of the undamaged and the damaged frames have been reported in Table 2, and the results have been compared to those obtained by means of the FEM model.

Analogously, the modal characteristics of the frames have been investigated. In particular, the first five frequencies of the undamaged and the damaged frames have been reported in Table 3 , and the results have been compared to those obtained by means of the FEM model.

These results show the accuracy of the proposed procedure.

\section{Damage Identification Procedure}

In this section, the damage parameters, that is, location and intensity, are evaluated by means of an optimization strategy based on the use of a certain number $n_{f}$ of frequencies of vibration of the considered frame.
For each possible damage location $k$ and for each damage intensity $j$, an opportune fitness function is defined as follows:

$$
F_{k, j}=F_{\max }-\sum_{i=1}^{n f}\left|\frac{\omega_{i}-\omega_{i, k j}}{\omega_{i u}}\right|, \quad j=1, \ldots, n_{i},
$$

where $F_{\max }$ is an arbitrary constant, chosen great enough to have always $F_{k, j}>0, n_{f}$ is the number of considered natural frequencies, $\widehat{\omega}_{i}$ is the measured $i$-th frequency of vibration, $\omega_{i, k j}$ is the calculated $i$-th frequency of vibration with intensity damage $j$ located in critical section $k$, and $\omega_{i u}$ is the $i$ th frequency of vibration for the undamaged frame.

Of course, among all the fitness functions, the one related to the exact position and intensity will reach its maximum value.

If the main purpose is to find only the location of the damage, for each critical section, the maximum fitness among those associated with different intensity values is chosen; then, the optimization procedure is performed.

$$
F_{k}=\max \left(F_{k, j}\right)
$$

It is worth pointing out that the proposed procedure applies both for damage identification in symmetric and unsymmetrical frames. Anyway, in case of symmetric frames, it is not possible to discern between symmetric damage positions. However, in order to carry out maintenance and restoration interventions, this circumstance does not appear to be a major limitation as it allows us to drastically reduce the sections to be considered to two only. Anyway, if one needs to univocally identify the damaged section in a symmetric frame, some information on mode shapes could be added in the objective function.

For symmetric frames, a reduced number $\bar{N}_{d}$ of critical sections can be defined as follows:

$$
\bar{N}_{d}=\left\{\begin{array}{ll}
\frac{N_{d}}{2}, & \text { if } N_{c} \text { is even } \\
\frac{N_{d}}{2}+N_{f}, & \text { if } N_{c} \text { is odd }
\end{array} .\right.
$$

\section{Numerical Applications}

In this section, the proposed damage identification procedure is applied to frames of different size. For each analysed frame, the frequencies of vibration of the undamaged and 


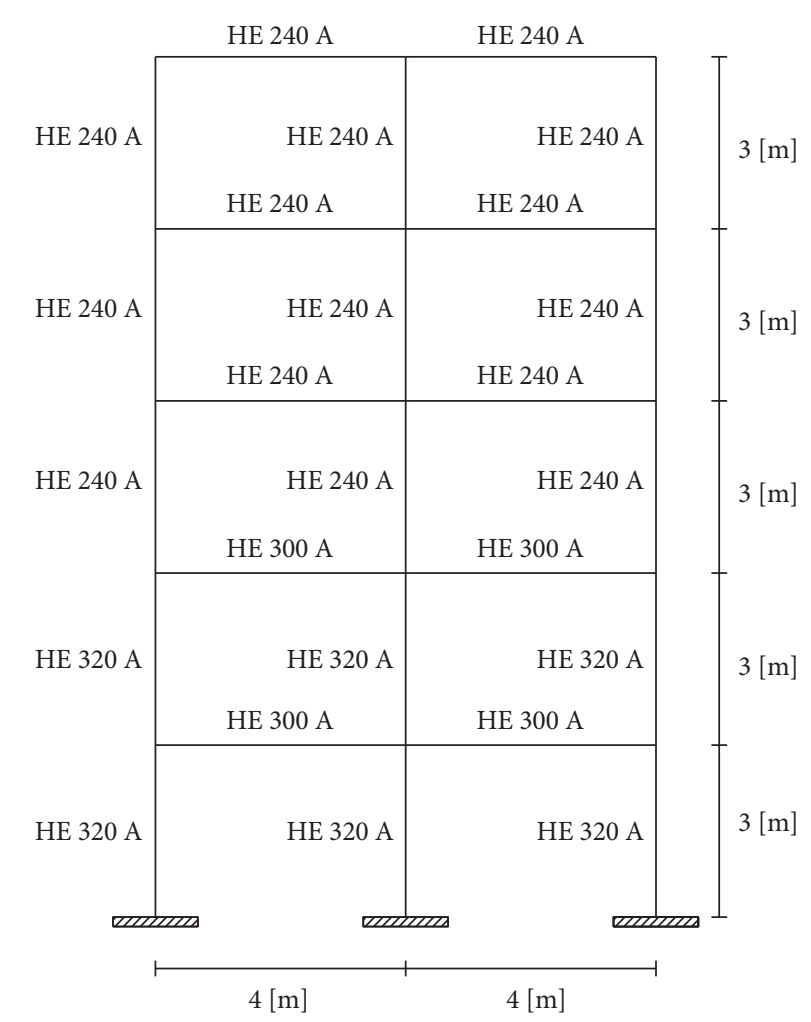

FIgURE 3: Frame with five floors and three columns.

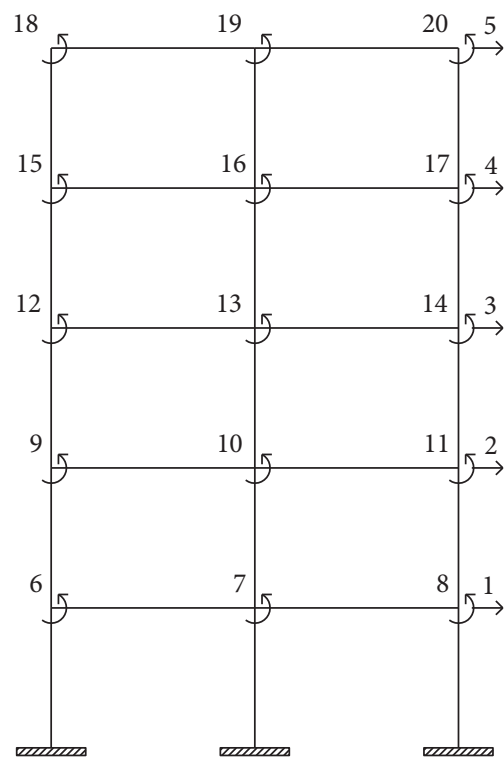

(a)

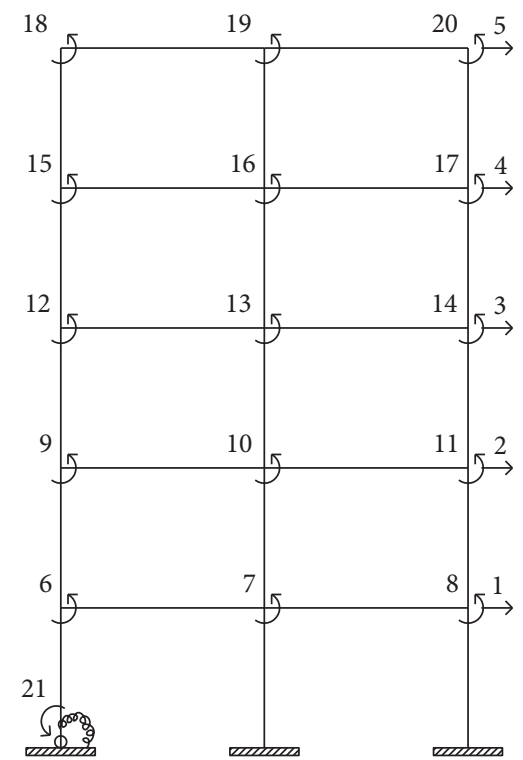

(b)

Figure 4: Degrees of freedom of the (a) undamaged and (b) damaged frame.

TABLE 1: Characteristics of the structural elements.

\begin{tabular}{lccc}
\hline Profile & $I_{x}\left(\mathrm{~m}^{4}\right)$ & $M_{\mathrm{y}} / \sigma_{y}\left(\mathrm{~m}^{3}\right)$ & $\mathrm{m}\left(\mathrm{kN}^{*} \mathrm{~s}^{2} / \mathrm{m}^{2}\right)$ \\
\hline HE320A & $22930 \cdot 10^{-8}$ & $1479 \cdot 10^{-6}$ & $97.6 \cdot 10^{-3}$ \\
HE300A & $18260 \cdot 10^{-8}$ & $1260 \cdot 10^{-6}$ & $88.3 \cdot 10^{-3}$ \\
HE240A & $7763 \cdot 10^{-8}$ & $675.1 \cdot 10^{-6}$ & $60.3 \cdot 10^{-3}$ \\
\hline
\end{tabular}


TABLe 2: Floor displacements of undamaged and damaged frames.

\begin{tabular}{lcccc}
\hline \multirow{2}{*}{ Floor } & \multicolumn{2}{c}{ Undamaged frame } & \multicolumn{2}{c}{ Damaged frame } \\
& Proposed procedure $(\mathrm{cm})$ & FEM model $(\mathrm{cm})$ & Proposed procedure $(\mathrm{cm})$ & 6.30 \\
FEM model $(\mathrm{cm})$ \\
\hline 1 & 6.14 & 6.14 & 14.85 & 6.30 \\
2 & 14.67 & 14.67 & 27.75 & 27.85 \\
3 & 27.57 & 27.57 & 38.68 & 38.68 \\
4 & 38.50 & 38.50 & 44.68 & 44.68 \\
5
\end{tabular}

TABle 3: Frequencies of undamaged and damaged frames.

\begin{tabular}{lcccc}
\hline Frequency & \multicolumn{2}{c}{ Undamaged frame } & \multicolumn{2}{c}{ Damaged frame } \\
& Proposed procedure & FEM model & Proposed procedure & FEM model \\
\hline 1 & 35.48 & 35.47 & 95.40 & 35.39 \\
2 & 96.16 & 96.10 & 167.17 & 16.66 \\
3 & 167.64 & 167.37 & 263.94 & 263.15 \\
4 & 264.53 & 263.74 & 351.27 & 349.81 \\
5
\end{tabular}

damaged configurations are previously evaluated. In particular, $N_{d}$ sets of $S_{d}$ frequencies are calculated, each one corresponding to a fixed location of the damage.

The availability of the algorithm developed in the present paper for the evaluation of the natural frequencies of frames of arbitrary sizes in the presence of single damage allows us to easily generate the data that must be compared to the experimentally measured ones in order to identify damage parameters. Some examples of location and intensity of the damage in the considered frames will be developed, and the related natural frequencies will be used as pseudoexperimental data in the identification procedure. Furthermore, in order to calibrate the mechanical parameters used to characterize the constitutive material, an opportune optimization procedure is described.

In principle, all the natural frequencies of all the possible configurations of damaged frames can be calculated, and the correct location of the damage can be identified, maximizing the fitness functions (7) and (8). The numerical applications will show that, in order to identify the two damage parameters, that is, location and intensity, it is enough to take into account only a few numbers of natural frequencies. Several damaged configurations have been tested for the considered frames in order to verify if the proposed optimization procedure is able to correctly detect the supposed damage. As reported in (6), the intensity of the damage, related to the stiffness of the rotational spring, varies with the parameter $\alpha_{i}$; in all the numerical applications, the following values of $\alpha_{i}$ have been considered: 1000, 800, 700, 500, 300, 100, 50, and 25 .

It is worth pointing out that the difference between the frequencies of vibration of undamaged and damaged frames increases with the intensity of the damage.
The results of the numerical applications are reported in different sections and concern a small frame with two floors and three columns and frames whose size progressively increases of one floor and one span.

Finally, in the last section, an iterative procedure able to identify the damage parameters without the need to evaluate the fitness function in all the critical sections, therefore reducing the computational effort, is presented.

6.1. Frame with Two Floors and Three Columns. The first developed application refers to the steel frame reported in Figure 5. The degrees of freedom for the considered symmetric frame in its undamaged configuration are eight as shown in Figure 2(a). Commercial steel profiles are considered for beams and columns and indicated in Figure 5, which also reports the length of the structural members. In Table 4, moments of inertia and the ratio between elastic moments of the cross sections and the yield stress are reported together with the values of the distributed masses, corresponding to an average specific weight $\mu=7.8494\left[\mathrm{kN} / \mathrm{m}^{4}\left(s^{2}\right)\right]$.

With the aim of accurately calibrating the mechanical parameters of the structural model, that is, Young modulus $E$ and specific weight $\mu$, it is assumed that the first three frequencies of vibration evaluated for $E=210000000$ $\left[\mathrm{kN} / \mathrm{m}^{2}\right]$ and $\mu=7.8494\left[\mathrm{kN} / \mathrm{m}^{4}\left(s^{2}\right)\right]$ are used as pseudoexperimental values in the minimization of the objective function (3).

The natural frequencies of the undamaged frame have been evaluated by solving equation (2) and assuming the following values of mechanical parameters: 


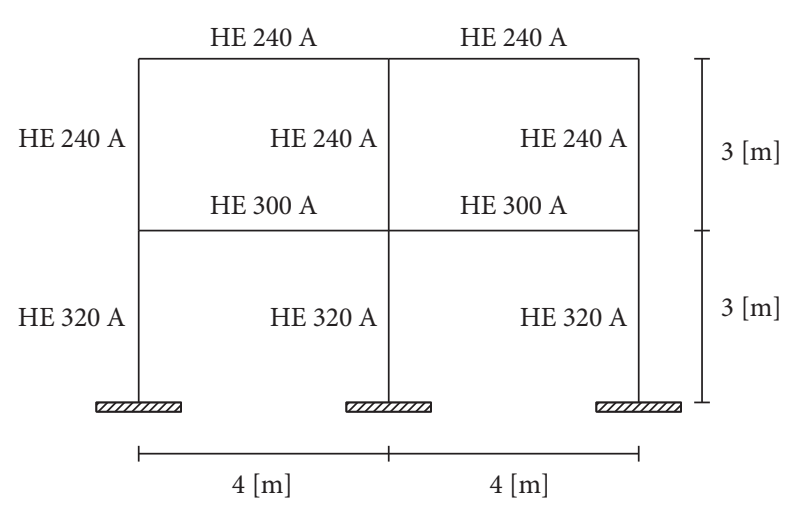

FIgURE 5: Frame with two floors and three columns.

TABLE 4: Characteristics of the structural elements.

\begin{tabular}{lccc}
\hline Profile & $I_{x}\left(\mathrm{~m}^{4}\right)$ & $M_{\mathrm{y}} / \sigma_{y}\left(\mathrm{~m}^{3}\right)$ & $\mathrm{m}\left(\mathrm{kN}^{*} \mathrm{~s}^{2} / \mathrm{m}^{2}\right)$ \\
\hline HE320A & $22930 \bullet 10^{-8}$ & $1479 \cdot 10^{-6}$ & $97.6 \cdot 10^{-3}$ \\
HE300A & $18260 \bullet 10^{-8}$ & $1260 \bullet 10^{-6}$ & $88.3 \cdot 10^{-3}$ \\
HE240A & $7763 \cdot 10^{-8}$ & $675.1 \cdot 10^{-6}$ & $60.3 \cdot 10^{-3}$ \\
\hline
\end{tabular}

$$
\begin{aligned}
& \widehat{E}=\left[\begin{array}{lllll}
200 & 206 & 210 & 215 & 220
\end{array}\right] \cdot 10^{6}\left[\frac{\mathrm{kN}}{\mathrm{m}^{2}}\right], \\
& \widehat{\mu}=\left[\begin{array}{llllllll}
6.2795 & 6.6720 & 7.0645 & 7.4569 & 7.8494 & 8.2419 & 8.6344 & 9.0268
\end{array}\right]\left[\frac{k N}{m^{4}} s^{2}\right] .
\end{aligned}
$$

For each value of the mechanical parameters $\widehat{\mathrm{E}} \widehat{\mu}$, the objective function (3) has been evaluated, showing the minimum value in correspondence of the assumed pseudoexperimental values (Figure 6).

In the following applications, the values $E=210000000\left[\mathrm{kN} / \mathrm{m}^{2}\right]$ and $\mu=7.8494\left[\mathrm{kN} / \mathrm{m}^{4}\left(s^{2}\right)\right]$ have

$$
\omega_{u}=\left[\begin{array}{lllll}
99,80 & 261,80 & 536,34 & 793,78 & 836,65
\end{array}\right.
$$

As already pointed out, the insertion of a hinge with a rotational spring to model the damage increases to nine degrees of freedom of the damaged frames and therefore the size of the stiffness and mass matrices. Since the frame is symmetric, $\bar{N}_{d}=11$ critical sections must be analysed; these have already been shown in Figure 2(b).

For each location and intensity of the damage, the nine frequencies have been evaluated by means of equation (5); these values are not here reported for the sake of brevity but will be used in the damage identification procedure.

For this frame, 9 natural frequencies can be calculated and considered as pseudoexperimental data. It will be shown that, in order to solve the inverse problem, it is enough to take into account 4 natural frequencies in the evaluation of the fitness functions related to each critical section. been used in the evaluation of the vibration properties for both undamaged and damaged frames.

The evaluation of the numerical values for the components of the stiffness and mass matrices allows us to solve the dynamic eigenvalue problem, which provides the following eight frequencies of vibration:

$\left.1,2346 \cdot 10^{3} \quad 1,3198 \cdot 10^{3} \quad 1,9954 \cdot 10^{3}\right][\mathrm{rad} / \mathrm{s}]$.

Assuming, for example, that the damage is located in the critical section 3 (Figure 2(b)) and has intensity represented by stiffness intensity parameter $\alpha=300$, the pseudoexperimental frequencies of vibration will therefore be assumed to be equal to

$$
\widehat{\omega}=\left[\begin{array}{llll}
99,76 & 261,64 & 536,20 & 793,14
\end{array}\right][\mathrm{rad} / \mathrm{s}] .
$$

Figure 7 reports the values that the fitness function would assume varying the location and intensity of the damage among the critical sections and clearly shows that the maximum value is reached in the correct location and intensity.

Another example can be shown; assuming that the damage is located in the critical section 7 (Figure 2(b)) and has intensity represented by stiffness intensity parameter 


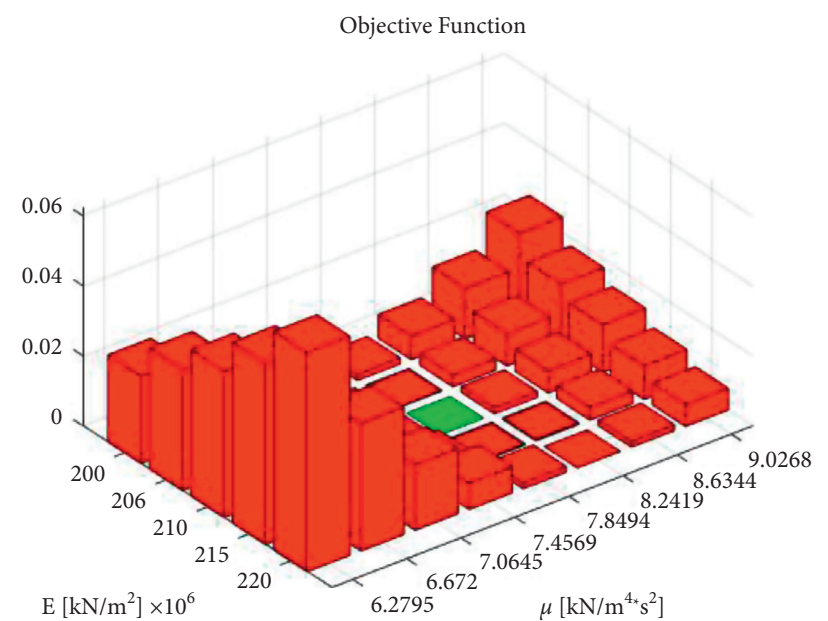

FIgURE 6: Calibration of mechanical parameters.

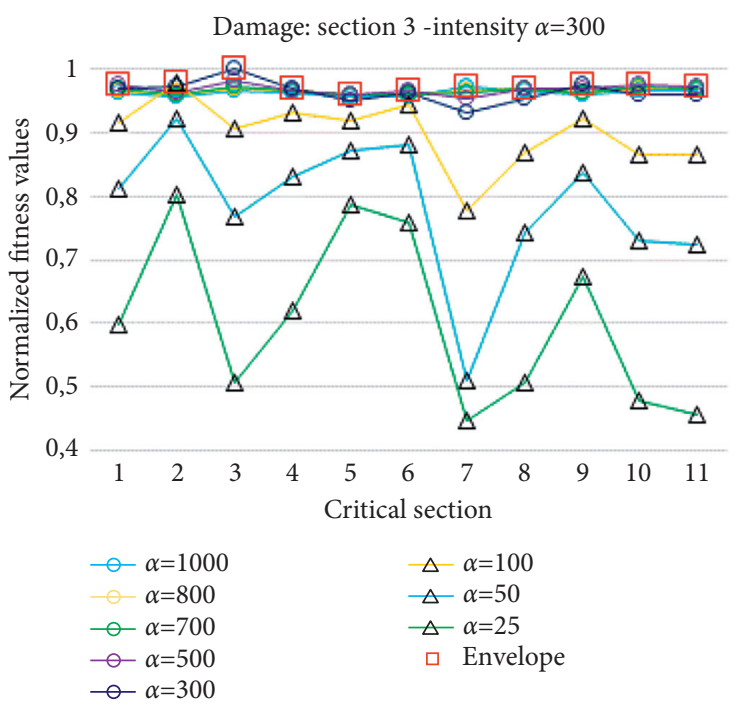

(a)

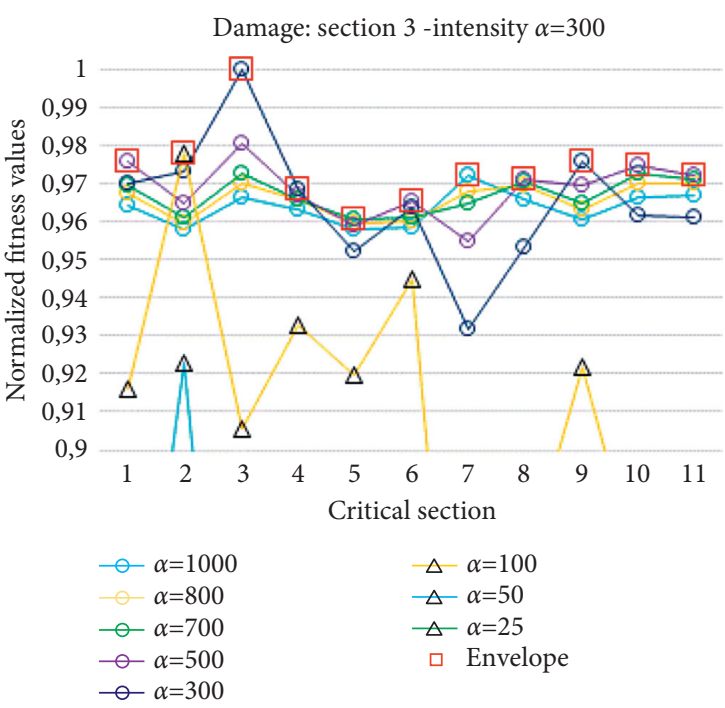

(b)

Figure 7: Normalized fitness values for damage with intensity $\alpha=300$ located in section 3 (a) and a zoom (b).

$\alpha=300$, the pseudoexperimental frequencies of vibration will therefore be assumed to be equal to

$$
\widehat{\omega}=\left[\begin{array}{llll}
99,78 & 261,74 & 536,21 & 791,25
\end{array}\right][\mathrm{rad} / \mathrm{s}] .
$$

Figure 8 reports the values that the fitness function would assume varying the location and intensity of the damage among the critical sections, and also in this case, it clearly shows that the maximum value is reached in the correct location and intensity.

6.2. Frames with Increasing Size. In this section, frames with an increasing number of floors and columns are analysed. In particular, as shown in Figure 9, a reference frame modulus composed of two columns and one beam, having, respectively, profiles HE $320 \mathrm{~A}$ and HE $240 \mathrm{~A}$, is first considered. Then, other frames are obtained, adding one floor and one column to the previous one. Of course, different parametric studies could be performed assuming, for example, frames with the same number of columns but different floors or vice versa.

In this section, 6 frames are analysed and labeled according to their number of floors; the number of critical sections for increasing size of the frame is reported in $\mathrm{Ta}$ ble 5. Assuming the presence of one damage, the degrees of freedom of the frames, and therefore, the number of frequencies of vibration is also reported in the same table. For each of the considered frames with variable damage parameters, the frequencies of vibration have been calculated by means of equation (5).

The parametric study has been developed assuming for each of the considered frames two possible damage positions located, respectively, at the base of the left column and at the right end of the beam of the first span at the first floor. In 


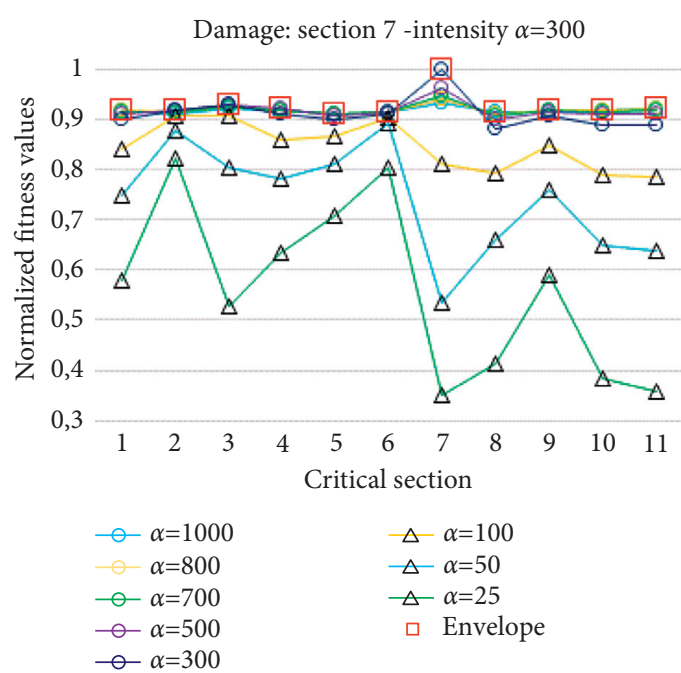

(a)

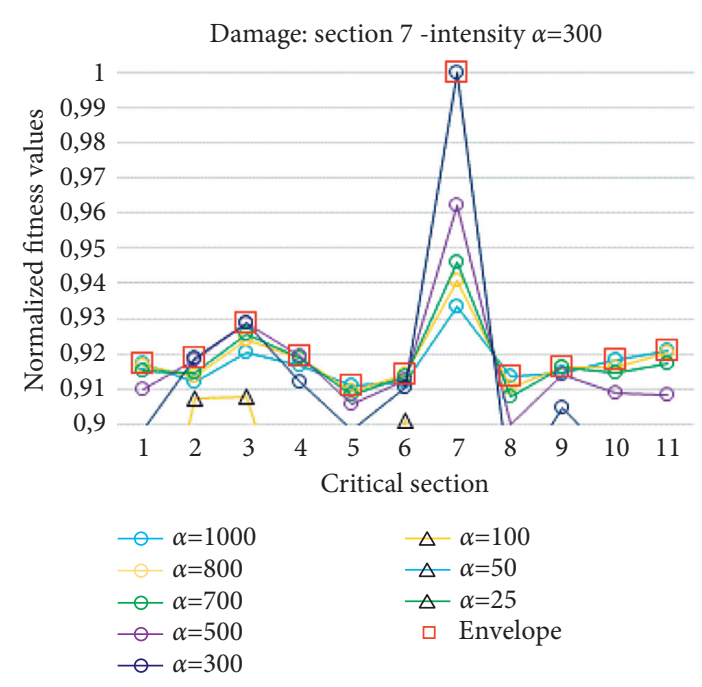

(b)

FIGURE 8: Normalized fitness values for damage with intensity $\alpha=300$ located in section 7 (a) and a zoom (b).

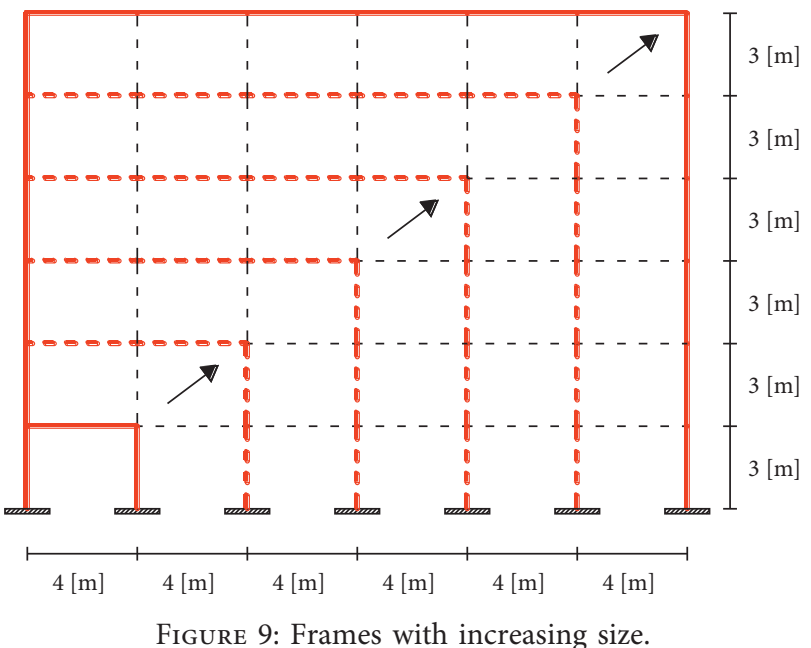

TABle 5: Characteristics of frames.

\begin{tabular}{lcc}
\hline No. of storeys & Critical sections & DOF \\
\hline 1 & 4 & 4 \\
2 & 18 & 9 \\
3 & 40 & 16 \\
4 & 70 & 25 \\
5 & 108 & 36 \\
6 & 154 & 49 \\
\hline
\end{tabular}

order to provide a clear representation of the obtained results, the critical sections have been renumbered as reported in Figure 10. It is worth noting that, since the analysed frames are symmetrical, the number of the considered critical sections is given by equation (9). As it can be easily noticed, the considered damaged sections are labeled as 1 and 4 for all the analysed frames.
Assuming a damage intensity represented by stiffness intensity parameter $\alpha=300$, it is possible to correctly identify both the damage location and intensity in all the analysed frames. In particular, the normalized maximum values of the fitness function for each critical section (Eq. 8) are reported for each frame in Figures 11 and 12, allowing us to easily identify the correct location of the damage.

6.3. An Iterative Algorithm for Damage Identification. In the previous section, it has been shown how the number $N_{d}$ of critical sections increases with the size of the frame. Since the proposed identification algorithm requires the calculation of a number of fitness functions equal to $n_{i} \times N_{d}$, it could be useful to investigate the possibility of obtaining reliable estimates of the damage parameters reducing the number of calculated fitness functions.

To this aim, an iterative identification algorithm has been realized within the software environment NetLogo [56] and presented in this section.

A generic frame can be reproduced in a virtual metric space, as shown in Figure 13. The critical sections $S_{\mathrm{k}}(k=1$, ..., 88 for the shown example of frame having five floors and five columns) are represented by yellow squares. Among them, let us call $S_{\mathrm{T}}$ the target (damaged) section, which in this example is placed at the bottom-left corner of the frame (indicated by a small concentric circle).

The identification procedure can be easily implemented by creating a "jumper" (green circle), able to move from one section to another, starting at $t=0$ from a given randomly selected section $S_{\mathrm{i}}$ on the frame (see panel (a) of Figure 13), for which the fitness $F_{\mathrm{i}}$ is calculated. Then, the algorithm goes on according to the following subsequent steps:

(1) At each iteration, the jumper explores the frame by calculating the fitness values associated with all the neighbouring sections included within an "exploration circle" of a given radius $R$ (chosen as 


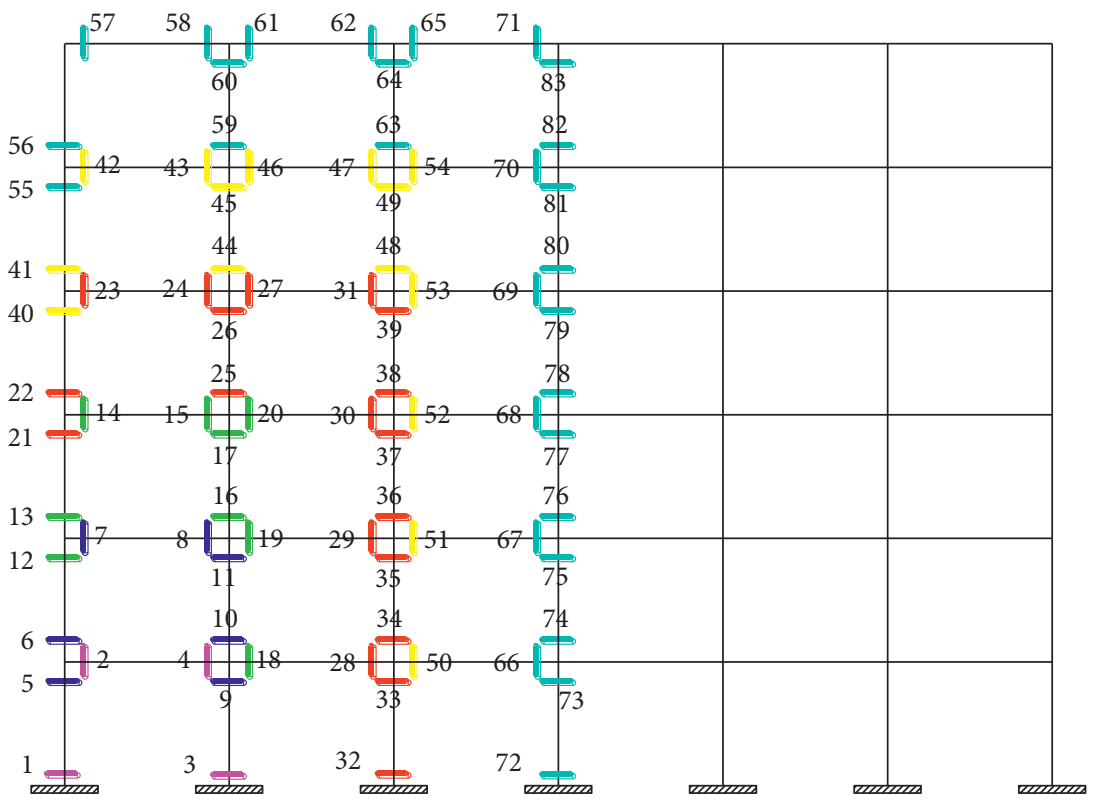

FIGURE 10: Renumbering of critical sections.

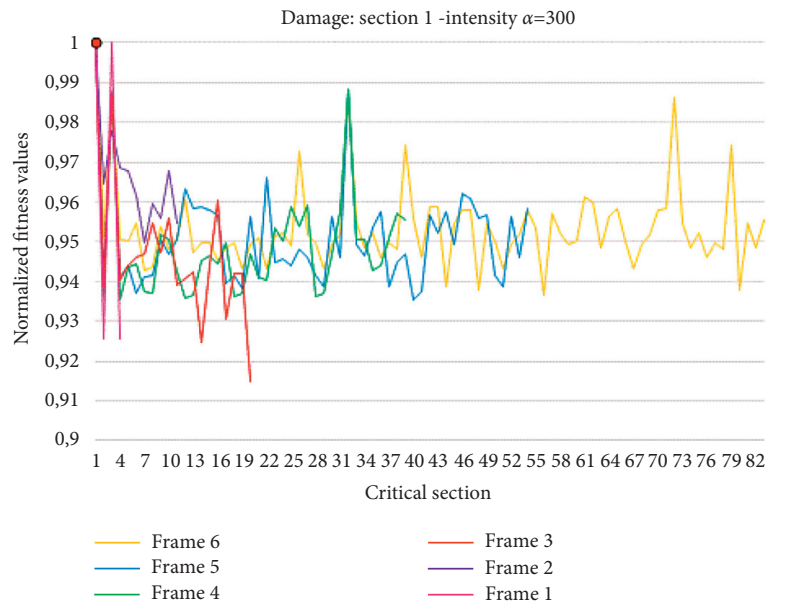

(a)

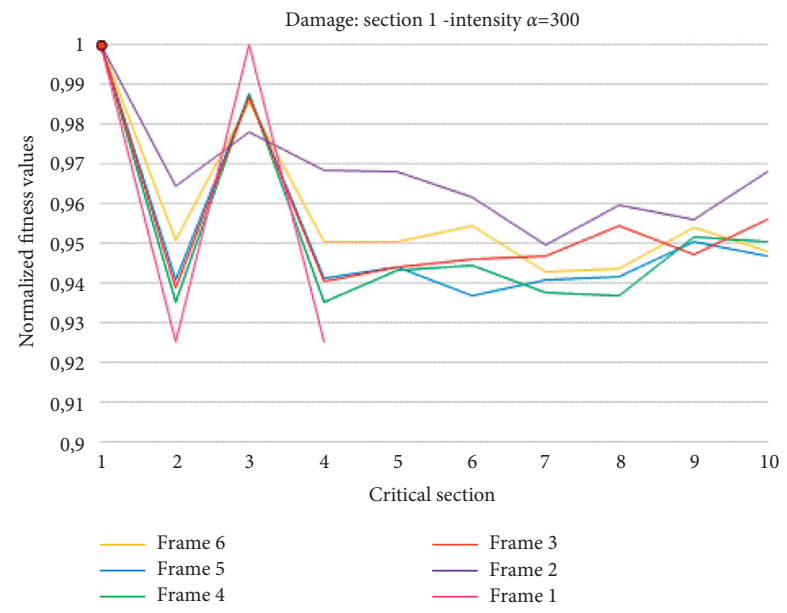

(b)

Figure 11: Normalized fitness values for damage with intensity $\alpha=300$ located in section 1 (a) and a zoom (b) for all the analysed frames.

equal to the distance between two floors). Sections for which the fitness has been calculated are coloured in red.

(2) Then, two options are available for the jumper:

(i) If some of these neighbouring sections have a fitness $F_{\mathrm{k}}>F_{\mathrm{i}}$, the jumper moves on the section with the maximum fitness among them.

(ii) If all the neighbouring sections have a fitness $F_{\mathrm{k}}<F_{\mathrm{i}}$, with a certain probability $0<p_{m}<1$ (called "moving probability," which introduces in the system a sort of noise useful to avoid local maxima), the jumper is forced to move on one of those sections, chosen at random; otherwise, the procedure is stopped.
(3) If the procedure has not been stopped at step 2, the algorithm comes back to step 1 and prosecutes (see panels (b) and (c)) until it is stopped. Notice that a given section can be considered more than one time during the entire procedure, but of course, its fitness is calculated only the first time the section has been included in the exploration circle.

(4) If the section reached by the jumper when it stops coincides with the target section $S_{\mathrm{T}}$, we say that the run has been successful.

In Figure 13(d), the situation at the end of a generic successful run is reported, with the jumper over the target. In this example, the percentage of explored (red) sections over the total is visibly less than $50 \%$, and this tells us that the 


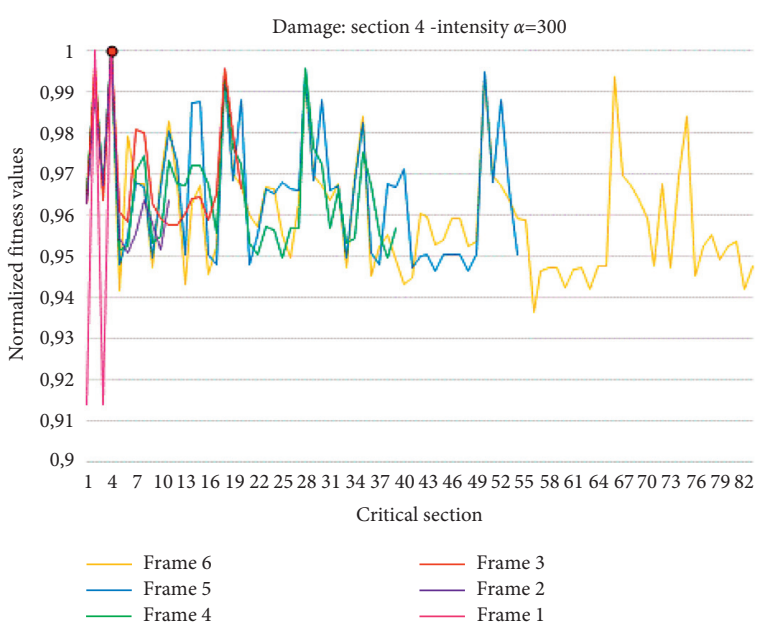

(a)

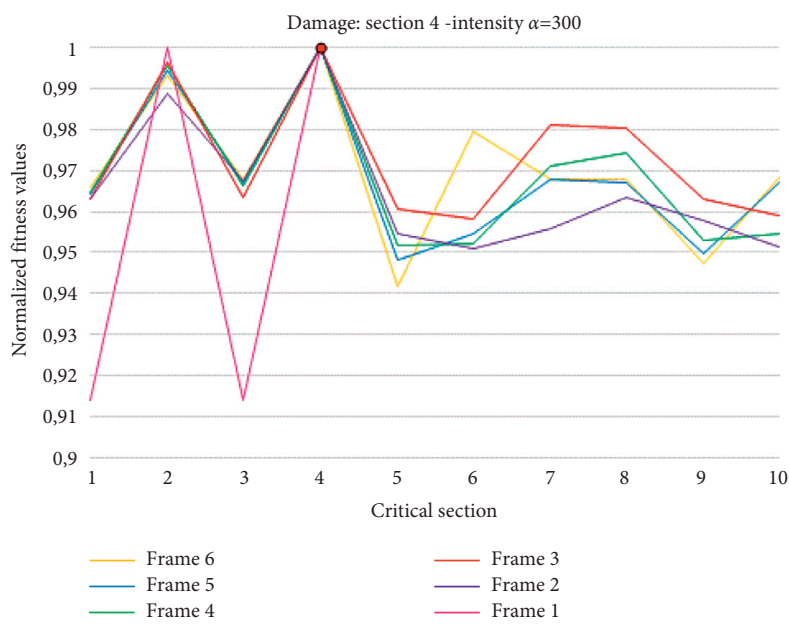

(b)

FIgURE 12: Normalized fitness values for damage with intensity $\alpha=300$ located in section 4 (a) and a zoom (b) for all the analysed frames.

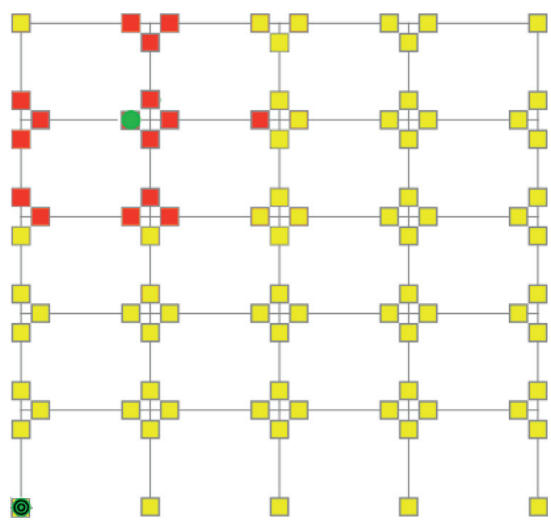

(a)

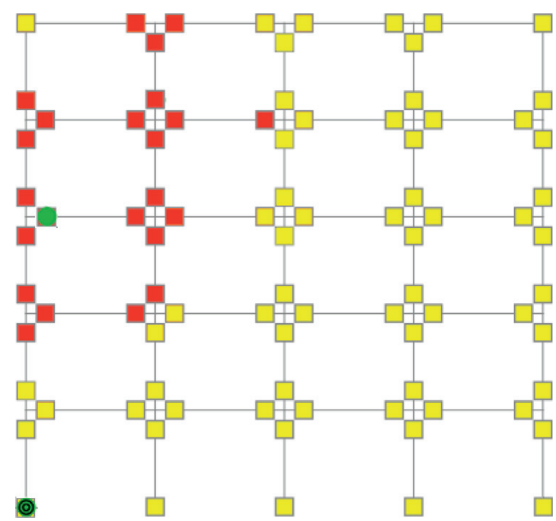

(b)

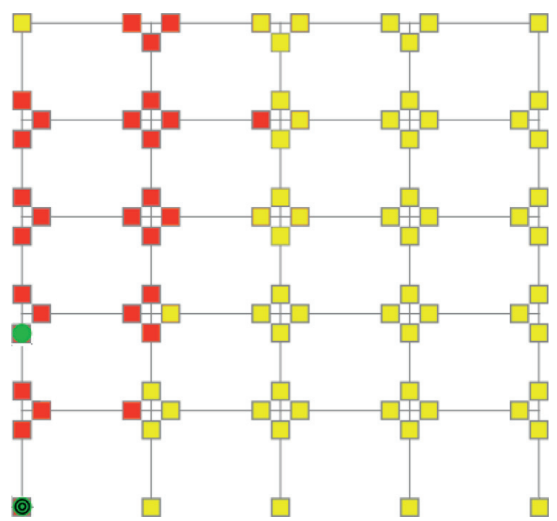

(c)

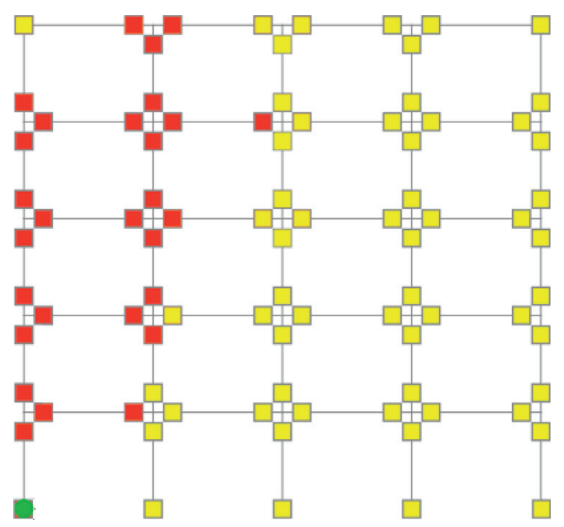

(d)

FIGURE 13: An example of the identification procedure for a frame with five floors and five columns. Starting from its initial position on a randomly selected section (a), the jumper (green circle) progressively explores its neighbouring sections (coloured in red) and moves on those with the best fitness $(b-c)$ until it reaches the target at the end of the run. See text for more details.

algorithm was not only effective (since the jumper reached the target) but also efficient, since we were able to identify the damaged section without needing to calculate the fitness for all the sections.
In order to have more reliable information about the effectiveness and the efficiency of the proposed algorithm for the identification of a given (fixed) target section, it is convenient to repeat $N$ times (with $N \gg>1$ ) the entire 

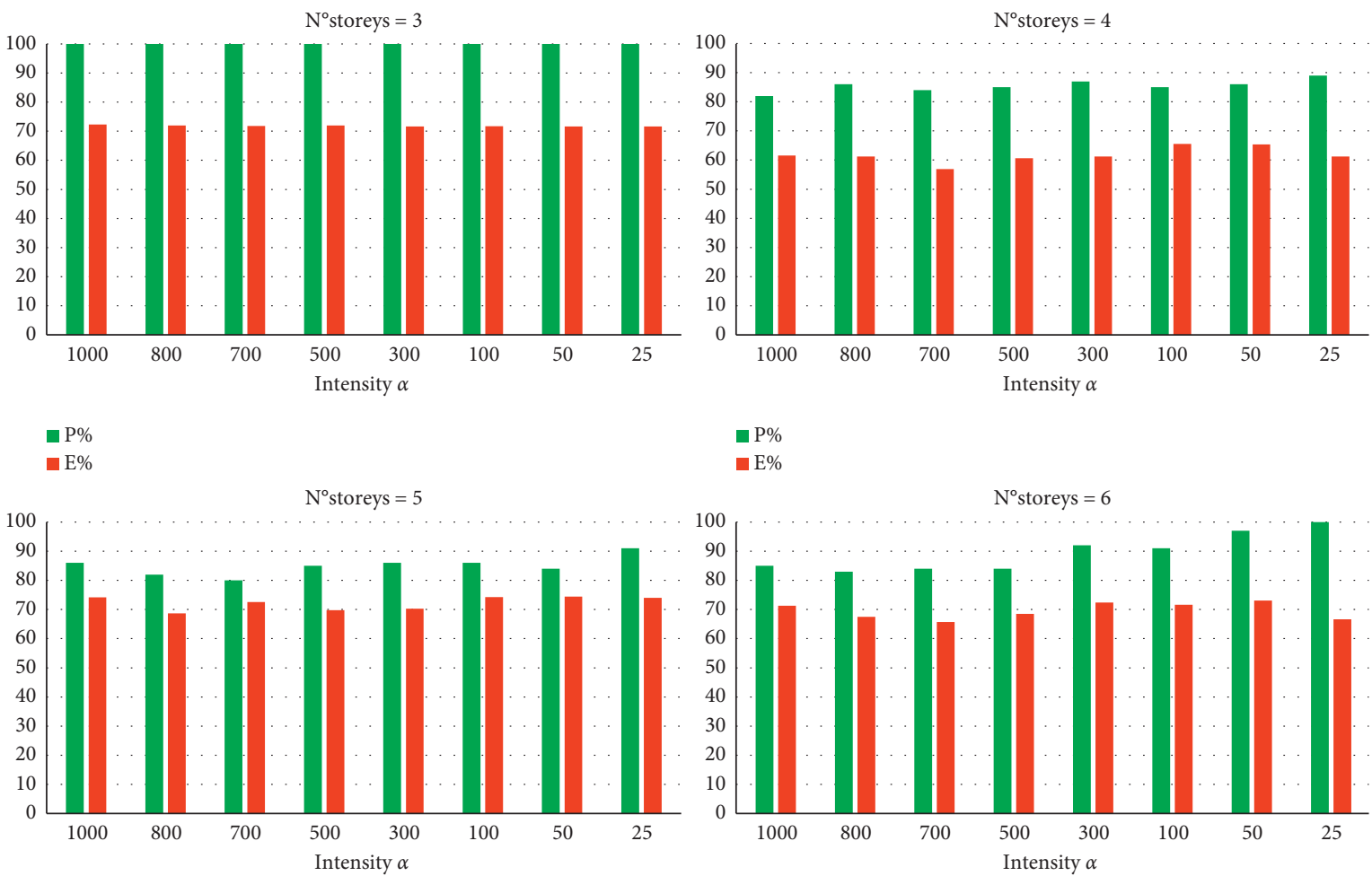

$$
\square \mathrm{P} \%
$$

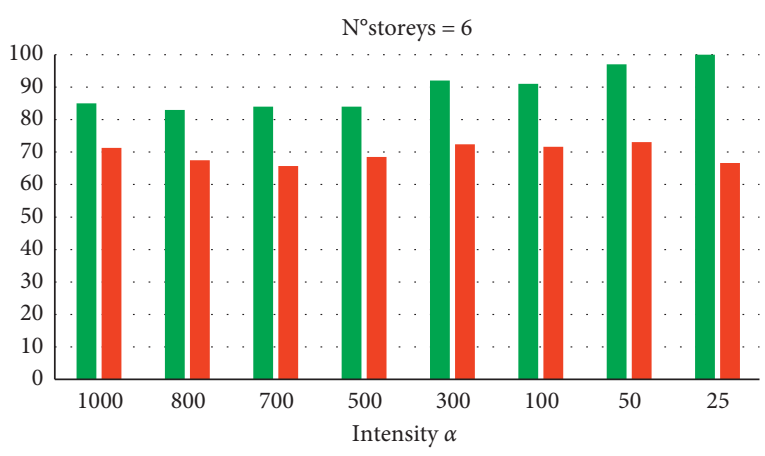

$\mathrm{P} \%$

- $\mathrm{P} \%$

- $\mathrm{E} \%$

FIgURE 14: P\% and E\% for each analysed frame with assigned damage location 1 and variable intensity.
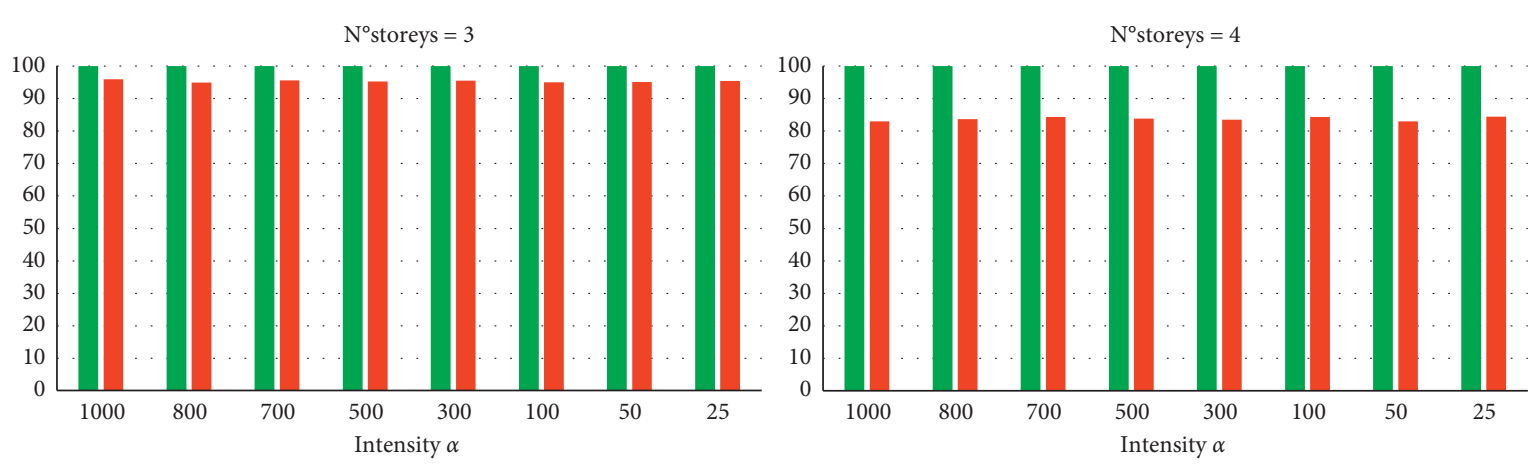

$$
\square \mathrm{P} \%
$$$$
\square \mathrm{P} \%
$$
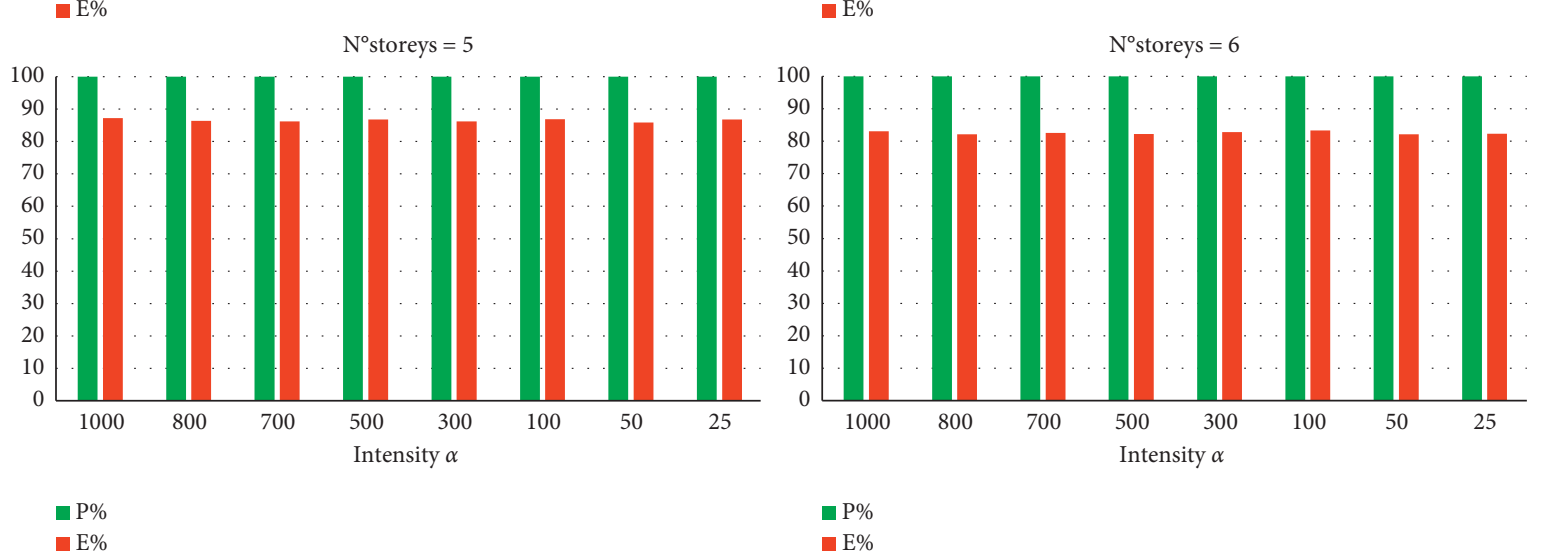

$\mathrm{P} \%$

FIgURE 15: P\% and E\% for each analysed frame with assigned damage location 4 and variable intensity. 


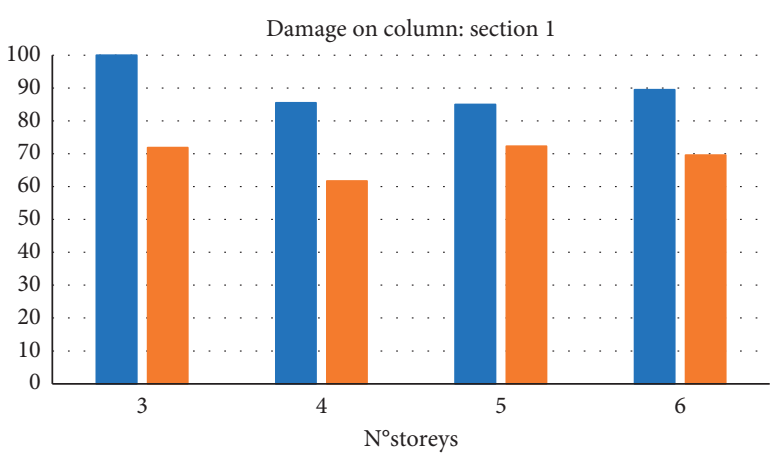

- $<\mathrm{P} \%>$

- $<\mathrm{E} \%>$

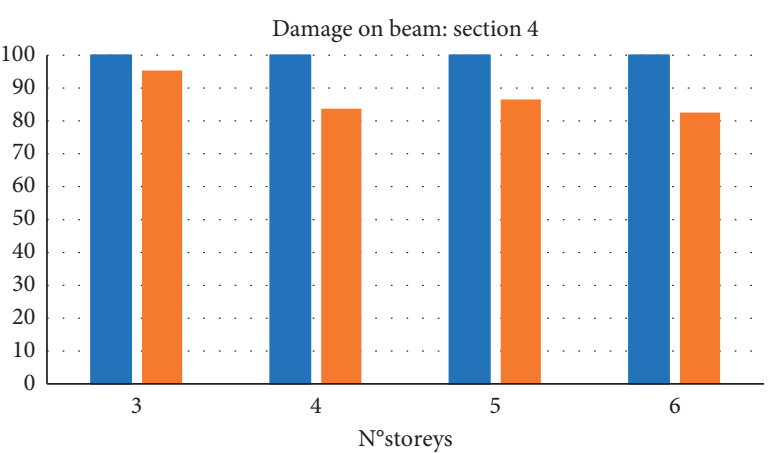

$-<\mathrm{P} \%>$

$<\mathrm{E} \%>$

(a)

(b)

FigURE 16: Mean values $\langle P \%>$ and $\langle E \%>$ calculated over the damage intensities $\alpha$ for increasing sizes of the frame: (a) damage location on column section 1; (b) damage location on beam section 4 .

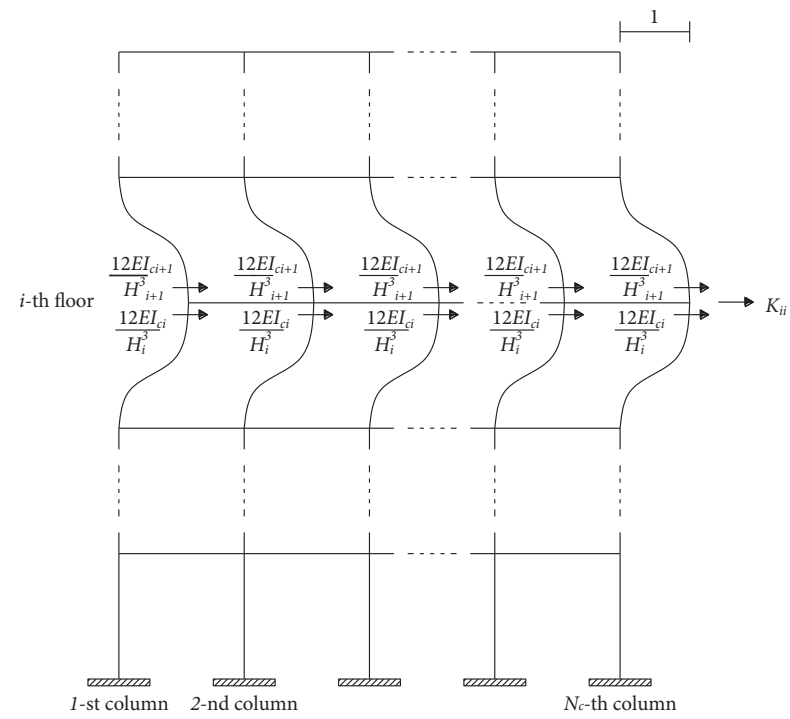

FIGURE 17: Scheme of the forces due to a unitary displacement at the (i)-th floor for computing the $K_{i i}$ term of the stiffness matrix.

procedure starting, for each run, from a different, randomly chosen section. At the end of the $N$ repetitions, the percentage $P \%$ of successful runs and the percentage $E \%$ of explored sections averaged over the $N$ runs will represent the quantities that we have to take into account for evaluating the performance of the algorithm. Of course, it would be desirable to maximize $\mathrm{P} \%$ and minimize $E \%$.

Let us now apply our iterative algorithm to the damage identification on several frames with increasing size, like those presented in paragraph 6.2. In particular, we limit our analysis to frames with a number of storeys greater than 2.

Assuming damage with intensity $\alpha$ located at the base of the left column (section 1 in Figure 10) or on a beam (section 4 in Figure 10), the average percentage P\% of successful runs and the average percentage $E \%$ of explored sections have been reported for each frame as a function of $\alpha$ in Figure 14

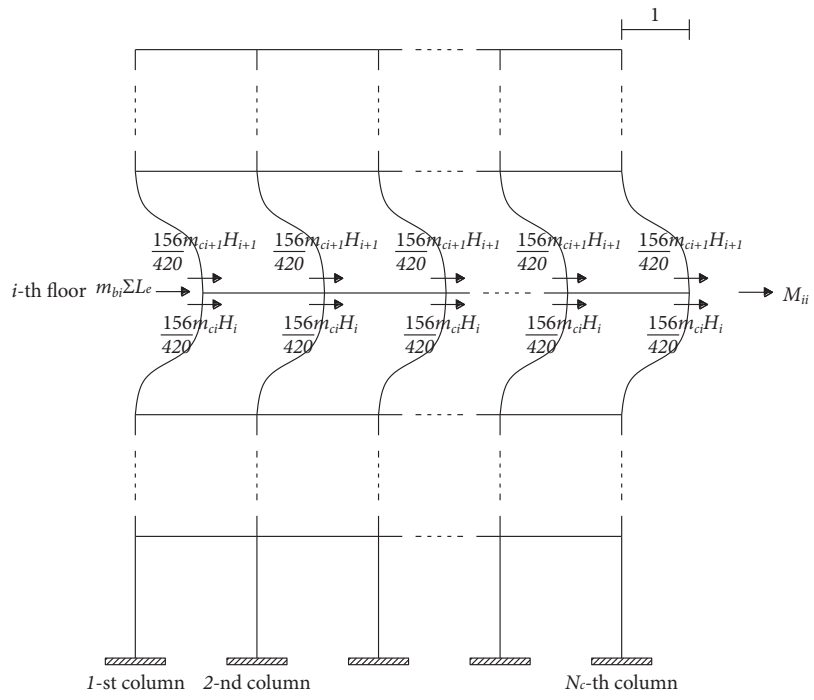

FIgURE 18: Scheme of the forces due to a unitary acceleration at the (i)-th floor for computing the $M_{i i}$ term of the mass matrix.

and 15, respectively. At first sight, it appears that, independently of $\alpha$, the algorithm seems more effective in finding the damaged section when the latter is located on a beam (location 4); however, in terms of explored sections, the procedure seems to perform better when the damaged section is located on the bottom-left column (location 1). To characterize this difference in a more quantitative way, in Figure $16(\mathrm{a})$, the mean values $\langle P \%\rangle$ and $\langle E \%\rangle$, averaged over the damage intensities $\alpha$ for each of the four frames, are reported for the damage section on location $1:\langle P \%\rangle$ decreases from $100 \%$, observed for a 3-storey frame, to values oscillating between $80 \%$ and $90 \%$ for larger frames, while $<E$ $\%>$ remains always included in the range $60 \%-70 \%$. For comparison, in Figure 16(b), the mean values $<P \%>$ and $<E$ $\%>$ are also reported for the damage section on location 4 : now $<P \%>$ stays constant at $100 \%$ for all frames, while $<E \%$ $>$ oscillates around $87 \%$. All the reported values seem to 


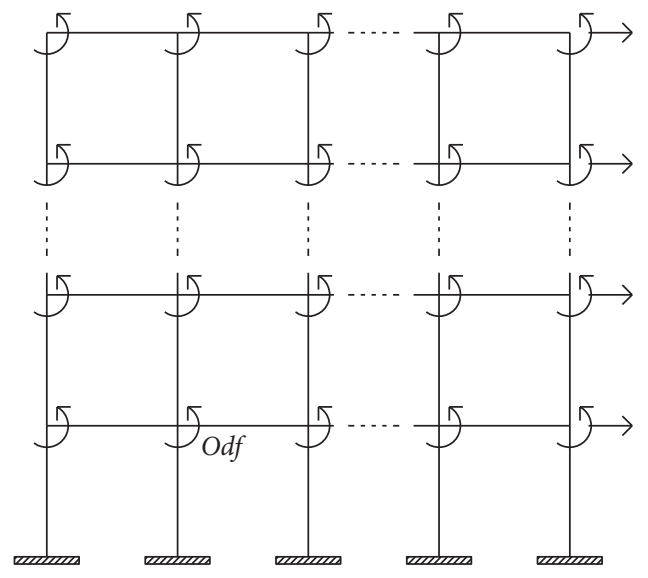

(a)

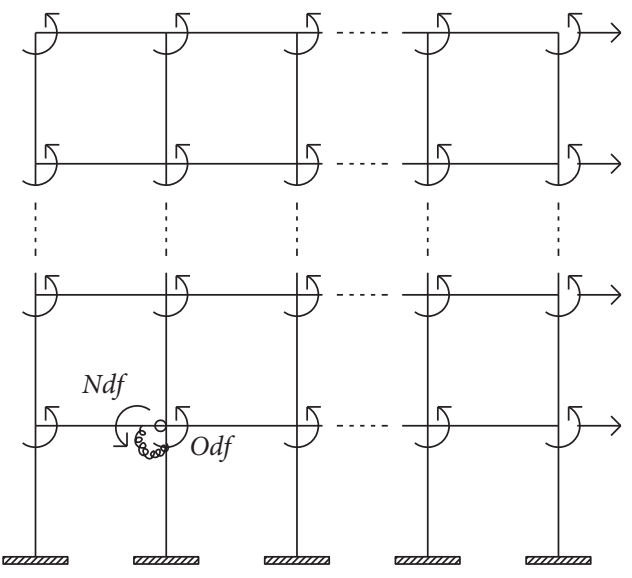

(b)

Figure 19: $O d f$ and $N d f$ in case of the undamaged (a) and damaged (b) frame.

confirm the utility of the presented iterative algorithm in finding reliable results reducing the needed computational effort.

\section{Conclusions}

The paper proposes an original algorithm for the evaluation of frequencies of vibration of frames with arbitrary numbers of columns and floors. The aim of the study is to apply the proposed algorithms for the solution of two optimization procedures concerning, respectively, the identification of the mechanical parameters of the constitutive material and the location and intensity of the first occurring damage. The damage induces a reduction in the bending stiffness and has been modeled by means of a hinge with rotational spring whose rigidity decreases with the intensity of the damage. All the joints between beams and columns have been considered as critical sections in which the damage can be localized.

The results in terms of frequencies of vibration and static displacements obtained by means of the proposed numerical models of undamaged and damaged frames have been compared to the correspondent values provided by an FEM model implemented in SAP 2000, showing a very good agreement.

The adopted optimization procedure aims at maximizing an opportune fitness function, which takes into account the numerical and experimental frequencies of vibration of the considered frame.

An original iterative procedure, which allows us to converge to the exact damage position without evaluating all the fitness functions, has been tested by means of several numerical applications referred to frames of different size, and the results show that it is possible to identify reliable damage parameters with a reduced computational burden.

\section{Appendix}

\section{A. Terms of the Stiffness Matrix of the Undamaged Frame}

The terms of the stiffness matrix have been determined by means of standard methods, which identify the terms of the matrix with the elastic nodal forces correspondent to unitary displacements of the degrees of freedom [57]. In Figure 17, by way of example, the term $K_{i i}$ that corresponds to the force at the $i$-th degree of freedom when a unitary displacement is assigned at the same degree of freedom is shown.

The stiffness matrix is reported in the following:

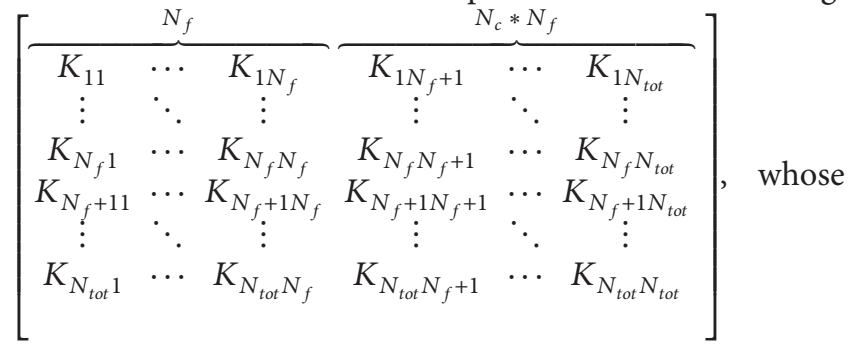

terms are

$$
\begin{aligned}
K_{i i} & =N_{c} \cdot 12 \frac{E I_{c, i}}{H_{i}^{3}}+N_{c} \cdot 12 \frac{E I_{c, i+1}}{H_{i+1}^{3}} \quad i=1: N_{f}-1, \\
K_{i i+1} & =-N_{c} \cdot 12 \frac{E I_{c, i+1}}{H_{i+1}^{3}} \quad i=1: N_{f}-1, \\
\text { For } i & =1: N_{f}-1,
\end{aligned}
$$




$$
\begin{aligned}
& K_{i j}=6 \frac{E I_{c, i}}{H_{i}^{2}}-6 \frac{E I_{c, i+1}}{H_{i+1}^{2}} \quad e=1: N_{c} ; \quad j=N_{f}+N_{c} \cdot(i-1)+e, \\
& K_{i j+N_{c}}=-6 \frac{E I_{c, i+1}}{H_{i+1}^{2}} \quad e=1: N_{c} ; \quad j=N_{f}+N_{c} \cdot(i-1)+e, \\
& K_{i+1 j}=6 \frac{E I_{c, i+1}}{H_{i+1}^{2}} \quad e=1: N_{c} ; \quad j=N_{f}+N_{c} \cdot(i-1)+e, \\
& K_{N_{f} N_{f}}=N_{c} \cdot 12 \frac{E I_{c, N_{f}}}{H_{N_{f}}^{3}}, \\
& K_{N_{f} j}=6 \frac{E I_{c, N_{f}}}{H_{N_{f}}^{2}} \quad e=1: N_{c} ; \quad j=N_{f}+N_{c} \cdot\left(N_{f}-1\right)+e, \\
& \text { For } s=0: N_{f}-2 \text {, } \\
& K_{i i}=4 \frac{E I_{c, s+1}}{H_{s+1}}+4 \frac{E I_{c, s+2}}{H_{s+2}}+4 \frac{E I_{b, s+1}}{L_{1}} \quad i=N_{f}+1+s \cdot N_{c}, \\
& K_{i i}=4 \frac{E I_{c, s+1}}{H_{s+1}}+4 \frac{E I_{c, s+2}}{H_{s+2}}+4 \frac{E I_{b, s+1}}{L_{e-1}}+4 \frac{E I_{b, s+1}}{L_{e}} \quad e=2: N_{c}-1 ; \quad i=N_{f}+e+s \cdot N_{c} \text {, } \\
& K_{i i}=4 \frac{E I_{c, s+1}}{H_{s+1}}+4 \frac{E I_{c, s+2}}{H_{s+2}}+4 \frac{E I_{b, s+1}}{L_{N_{c}-1}} \quad i=N_{f}+N_{c}+s \cdot N_{c} \text {, } \\
& K_{i i}=4 \frac{E I_{c, N_{f}}}{H_{N_{f}}}+4 \frac{E I_{b, N_{f}}}{L_{1}} \quad i=N_{t o t}-N_{c}+1 \text {, } \\
& K_{i i}=4 \frac{E I_{c, N_{f}}}{H_{N_{f}}}+4 \frac{E I_{b, N_{f}}}{L_{e-1}}+4 \frac{E I_{b, N_{f}}}{L_{e}} \quad e=2: N_{c}-1 ; \quad i=N_{t o t}-N_{c}+e \text {, } \\
& K_{i i}=4 \frac{E I_{c, N_{f}}}{H_{N_{f}}}+4 \frac{E I_{b, N_{f}}}{L_{N_{c}-1}} \quad i=N_{t o t} \text {, } \\
& K_{i j}=2 \frac{E I_{c, s+1}}{H_{s+1}} \quad e=1:\left(N_{f}-1\right) \cdot N_{c} ; \quad s=\operatorname{ceil}\left(\frac{e}{N_{c}}\right) ; \quad i=N_{f}+e ; \quad j=N_{c}+i, \\
& \text { For } s=0: N_{f}-1 \text {, } \\
& K_{i i+1}=2 \frac{E I_{b, s+1}}{L_{e}} \quad e=1: N_{c}-1 ; \quad i=N_{f}+e+s \cdot N_{c},
\end{aligned}
$$

where $N_{\text {tot }}=N_{f}\left(1+N_{c}\right)$ is the total number of degrees of freedom.

The remaining half matrix is built for symmetry.

It is worth noting that the above-reported terms apply when the number of floors and spans of the frame is greater than one. If the frame has one floor and one span, the terms of the stiffness matrix are the following:

$$
\begin{aligned}
K_{11} & =24 \frac{E I_{c, 1}}{H_{1}^{3}}, \\
K_{12} & =6 \frac{E I_{c, 1}}{H_{1}^{2}}, \\
K_{13} & =6 \frac{E I_{c, 1}}{H_{1}^{2}}, \\
K_{22} & =4 \frac{E I_{c, 1}}{H_{1}}+4 \frac{E I_{b, 1}}{L_{1}},
\end{aligned}
$$

$$
\begin{aligned}
& K_{23}=2 \frac{E I_{b, 1}}{L_{1}}, \\
& K_{33}=4 \frac{E I_{c, 1}}{H_{1}}+4 \frac{E I_{b, 1}}{L_{1}} .
\end{aligned}
$$

The remaining half matrix is built for symmetry.

\section{B. Terms of the Mass Matrix of the Undamaged Frame}

The terms of the mass matrix have been determined by means of standard methods, which identify the terms of the matrix with the inertial nodal forces correspondent to the unitary acceleration of the degrees of freedom [57]. In Figure 18 , by way of example, the term $M_{i i}$ which corresponds to the force at the $i$-th degree of freedom when a unitary acceleration is assigned at the same degree of freedom is shown. 
The mass matrix is reported in the following:

$\left[\begin{array}{cccccc}\overbrace{M_{11}} & \cdots & M_{1 N_{f}} & M_{1 N_{f}+1} & \cdots & M_{1 N_{t o t}} \\ \vdots & \ddots & \vdots & \vdots & \ddots & \vdots \\ M_{N_{f} 1} & \cdots & M_{N_{f} N_{f}} & M_{N_{f} N_{f}+1} & \cdots & M_{N_{f} N_{t o t}} \\ M_{N_{f}+11} & \cdots & M_{N_{f}+1 N_{f}} & M_{N_{f}+1 N_{f}+1} & \cdots & M_{N_{f}+1 N_{t o t}} \\ \vdots & \ddots & \vdots & \vdots & \ddots & \vdots \\ M_{N_{t o t} 1} & \cdots & M_{N_{t o t} N_{f}} & M_{N_{t o t} N_{f}+1} & \cdots & M_{N_{t o t} N_{t o t}}\end{array}\right]$,

whose terms are

$$
\begin{aligned}
M_{i i} & =N_{c} \cdot \frac{156}{420} m_{c, i} H_{i}+N_{c} \cdot \frac{156}{420} m_{c, i+1} H_{i+1}+m_{b, i} \sum_{e=1}^{N c-1} L_{e} \quad i=1: N_{f}-1, \\
M_{i i+1} & =N_{c} \cdot \frac{54}{420} m_{c, i+1} H_{i+1} \quad i=1: N_{f}-1,
\end{aligned}
$$

For $\quad i=1: N_{f}-1$,

$$
\begin{aligned}
& M_{i j}=\frac{22}{420} m_{c, i} H_{i}^{2}-\frac{22}{420} m_{c, i+1} H_{i+1}^{2} \quad e=1: N_{c} ; \quad j=N_{f}+N_{c} \cdot(i-1)+e, \\
& M_{i j+N_{c}}=\frac{13}{420} m_{c, i+1} H_{i+1}^{2} \quad e=1: N_{c} ; \quad j=N_{f}+N_{c} \cdot(i-1)+e, \\
& M_{i+1 j}=-\frac{13}{420} m_{c, i+1} H_{i+1}^{2} \quad e=1: N_{c} ; \quad j=N_{f}+N_{c} \cdot(i-1)+e, \\
& M_{N_{f} N_{f}}=N_{c} \cdot \frac{156}{420} m_{c, N_{f}} H_{N_{f}}+m_{b, N_{f}} \sum_{e=1}^{N_{c}-1} L_{e} \\
& M_{N_{f} j}=\frac{22}{420} m_{c, N_{f}} H_{N_{f}}^{2} \quad e=1: N_{c} ; \quad j=N_{f}+N_{c} \cdot\left(N_{f}-1\right)+e, \\
& \text { For } s=0: N_{f}-2 \text {, } \\
& M_{i i}=\frac{4}{420} m_{c, s+1} H_{s+1}^{3}+\frac{4}{420} m_{c, s+2} H_{s+2}^{3}+\frac{4}{420} m_{b, s+1} L_{1}^{3} \quad i=N_{f}+1+s \cdot N_{c}, \\
& M_{i i}=\frac{4}{420} m_{c, s+1} H_{s+1}^{3}+\frac{4}{420} m_{c, s+2} H_{s+2}^{3}+\frac{4}{420} m_{b, s+1} L_{e-1}^{3}+\frac{4}{420} m_{b, s+1} L_{e}^{3} \quad e=2: N_{c}-1 ; \quad i=N_{f}+e+s \cdot N_{c}, \\
& M_{i i}=\frac{4}{420} m_{c, s+1} H_{s+1}^{3}+\frac{4}{420} m_{c, s+2} H_{s+2}^{3}+\frac{4}{420} m_{b, s+1} L_{N_{c}-1}^{3} \quad i=N_{f}+N_{c}+s \cdot N_{c} \\
& M_{i i}=\frac{4}{420} m_{c, N_{f}} H_{N_{f}}^{3}+\frac{4}{420} m_{b, N_{f}} L_{1}^{3} \quad i=N_{t o t}-N_{c}+1 \text {, } \\
& M_{i i}=\frac{4}{420} m_{c, N_{f}} H_{N_{f}}^{3}+\frac{4}{420} m_{b, N_{f}} L_{e-1}^{3}+\frac{4}{420} m_{b, N_{f}} L_{e}^{3} \quad e=2: N_{c}-1 ; \quad i=N_{t o t}-N_{c}+e, \\
& M_{i i}=\frac{4}{420} m_{c, N_{f}} H_{N_{f}}^{3}+\frac{4}{420} m_{b, N_{f}} L_{N_{c}-1}^{3} \quad i=N_{t o t} \text {, } \\
& M_{i j}=-\frac{3}{420} m_{c, s+1} H_{s+1}^{3} \quad e=1:\left(N_{f}-1\right) \cdot N_{c} ; \quad s=\operatorname{ceil}\left(\frac{e}{N_{c}}\right) ; \quad i=N_{f}+e ; \quad j=N_{c}+i,
\end{aligned}
$$

For $s=0: N_{f}-1$,

$$
M_{i i+1}=-\frac{3}{420} m_{b, s+1} L_{e}^{3} \quad e=1: N_{c}-1 ; \quad i=N_{f}+e+s \cdot N_{c},
$$


where $N_{t o t}=N_{f}\left(1+N_{c}\right)$ is the total number of degrees of freedom.

The remaining half matrix is built for symmetry.

It is worth noting that the above-reported terms apply when the number of floors and spans of the frame is greater than one. If the frame has one floor and one span, the terms of the mass matrix are the following:

$$
\begin{aligned}
& M_{11}=2 \cdot \frac{156}{420} m_{c, 1} H_{1}+m_{b, 1} L_{1}, \\
& M_{12}=\frac{22}{420} m_{c, 1} H_{1}^{2}, \\
& M_{13}=\frac{22}{420} m_{c, 1} H_{1}^{2}, \\
& M_{22}=\frac{4}{420} m_{c, 1} H_{1}^{3}+\frac{4}{420} m_{b, 1} L_{1}^{3}, \\
& M_{23}=-\frac{3}{420} m_{b, 1} L_{1}^{3}, \\
& M_{33}=\frac{4}{420} m_{c, 1} H_{1}^{3}+\frac{4}{420} m_{b, 1} L_{1}^{3} .
\end{aligned}
$$

The remaining half matrix is built for symmetry.

\section{Terms of the Stiffness and Mass Matrices of the Damaged Frame}

It is useful to denote the degree of freedom in the undamaged frame as $O d f$, which, in the absence of the hinge and the spring, would rotate the end of the structural member where the damage is located, as shown in Figure 19. In case of damage located at the base of a column of the first floor, $\mathrm{Odf}$ is the degree of freedom corresponding to the top of the column. All the terms of the stiffness and mass matrices of the undamaged frame remain the same except the ones related to the $O d f$ degree of freedom and to the new degree of freedom denoted as $N d f=S_{d}$, as shown in the following.

In particular, in order to modify the matrices, a different algorithm has been applied according to the position of the damage, that is, beam or column. For the sake of brevity, only the case of the stiffness matrix is described in the following. The algorithm for the mass matrix follows the same steps, just substituting $I_{b i}$ and $I_{c i}$ with $m_{b i}$ and $m_{c i}$, respectively, adopting the appropriate coefficients and neglecting the mass and stiffness of the rotational spring.

In case of damage located in a beam, the terms of the stiffness matrix change as in the following:

$$
\begin{aligned}
& K_{\text {OdfOdf }}^{d}=K_{\text {Odf Odf }}^{u}-\frac{4 E I_{b D}}{L_{D}}+k_{\varphi}, \\
& K_{N d f N d f}^{d}=\frac{4 E I_{b D}}{L_{D}}+k_{\varphi}, \\
& K_{\text {Odf Ndf }}^{d}=-k_{\varphi} .
\end{aligned}
$$

If the damage is located at the right end of the beam,

$$
\begin{aligned}
& K_{\text {Odf-1 Odf }}^{d}=0, \\
& K_{\text {Odf-1 Ndf }}^{d}=\frac{2 E I_{b D}}{L_{D}} .
\end{aligned}
$$

If the damage is located at the left end of the beam,

$$
\begin{aligned}
& K_{\text {Odf Odf }+1}^{d}=0, \\
& K_{\text {Odf+1 Ndf }}^{d}=\frac{2 E I_{b D}}{L_{D}},
\end{aligned}
$$

where $I_{b D}$ and $L_{D}$ denote, respectively, the moment of inertia and the length of the beam where the damage is located.

In case of damage located in a column, the terms of the stiffness matrix change as in the following:

$$
\begin{aligned}
& K_{\text {Odf Odf }}^{d}=K_{\text {Odf Odf }}^{u}-\frac{4 E I_{c D}}{H_{D}}+k_{\varphi}, \\
& K_{N d f N d f}^{d}=\frac{4 E I_{c D}}{H_{D}}+k_{\varphi}, \\
& K_{\text {Odf Ndf }}^{d}=-k_{\varphi} .
\end{aligned}
$$

If the damage is located at the top end of the column,

$$
\begin{aligned}
K_{\text {FlOdf }}^{d} & =K_{F l O d f}^{u}-\frac{6 E I_{c D}}{H_{D}^{2}}, \\
K_{F l N d f}^{d} & =\frac{6 E I_{c D}}{H_{D}^{2}}, \\
K_{F l-1 \text { Odf }}^{d} & =0 \text { with } F l>1, \\
K_{F l-1 N d f}^{d} & =-\frac{6 E I_{c D}}{H_{D}^{2}} \text { with } F l>1, \\
K_{\text {Odf-NcOdf }}^{d} & =0 \text { with } F l>1, \\
K_{\text {Odf-Nc Ndf }}^{d} & =\frac{2 E I_{c D}}{H_{D}} \text { with } F l>1 .
\end{aligned}
$$

If the damage is located at the bottom end of the column,

$$
\begin{aligned}
K_{F l O d f}^{d} & =0 \\
K_{F l N d f}^{d} & =\frac{6 E I_{c D}}{H_{D}^{2}}, \\
K_{F l-1 O d f}^{d} & =K_{F l-1 O d f}^{u}+\frac{6 E I_{c D}}{H_{D}^{2}}, \\
K_{F l-1 N d f}^{d} & =-\frac{6 E I_{c D}}{H_{D}^{2}}, \\
K_{O d f O d f+N c}^{d} & =0, \\
K_{O d f+N c N d f}^{d} & =\frac{2 E I_{c D}}{H_{D}} .
\end{aligned}
$$


If the damage is located at the bottom end of a column of the first floor, only the following terms change:

$$
\begin{aligned}
K_{1 N d f}^{d} & =\frac{6 E I_{c D}}{H_{D}^{2}}, \\
K_{N d f N d f}^{d} & =\frac{4 E I_{c D}}{H_{D}}+k_{\varphi}, \\
K_{\text {Odf Ndf }}^{d} & =\frac{2 E I_{c D}}{H_{D}},
\end{aligned}
$$

where $F l, I_{c D}$, and $H_{D}$ denote, respectively, the floor, the moment of inertia, and the length of the column where the damage is located.

The remaining half matrix is built for symmetry.

\section{Data Availability}

The data used to support the findings of this study are available from the corresponding author upon request.

\section{Conflicts of Interest}

The authors declare that they have no conflicts of interest.

\section{Acknowledgments}

This research was funded by the University of Catania, with the projects "Linea di Intervento 2 del Piano di Incentivi per la Ricerca di Ateneo PIACERI 2020/2022 " of the Departments of Civil Engineering and Architecture and Physics and Astronomy "Ettore Majorana."

\section{References}

[1] T. G. Chondros and A. D. Dimarogonas, "Dynamic sensitivity of structures to cracks," Journal of Vibration and Acoustics, vol. 111, no. 3, pp. 251-256, 1989.

[2] M.-H. H. Shen and C. Pierre, "Natural modes of BernoulliEuler beams with symmetric cracks," Journal of Sound and Vibration, vol. 138, no. 1, pp. 115-134, 1990.

[3] W. M. Ostachowicz and M. Krawczuk, "Analysis of the effect of cracks on the natural frequencies of a cantilever beam," Journal of Sound and Vibration, vol. 150, no. 2, pp. 191-201, 1991.

[4] I. Caliò, D. D’Urso, and A. Greco, "The influence of damage on the eigen-properties of Timoshenko spatial arches," Computers \& Structures, vol. 190, pp. 13-24, 2017.

[5] B. Bozyigit, Y. Yesilce, and M. A. Wahab, "Free vibration and harmonic response of cracked frames using a single variable shear deformation theory," Structural Engineering \& $\mathrm{Me}$ chanics, vol. 74, no. 1, pp. 33-54, 2020.

[6] K. Mazanoglu and E. C. Kandemir-Mazanoglu, "A new simplified method for anti-symmetric mode in-plane vibrations of frame structures with column cracks," Journal of Vibration and Control, vol. 24, no. 24, pp. 5794-5810, 2018.

[7] U. Eroglu and E. Tufekci, "Free vibration of damaged frame structures considering the effects of axial extension, shear deformation and rotatory inertia: exact solution," International Journal of Structural Stability and Dynamics, vol. 17, no. 10, 2017.
[8] F. Cannizzaro, A. Greco, S. Caddemi, and I. Caliò, "Closed form solutions of a multi-cracked circular arch under static loads," International Journal of Solids and Structures, vol. 121, pp. 191-200, 2017.

[9] A. M. Ibrahim, H. Ozturk, and M. Sabuncu, "Vibration analysis of cracked frame structures," Structural Engineering \& Mechanics, vol. 45, no. 1, pp. 33-52, 2013.

[10] M. Cerri and F. Vestroni, "Identification of damage due to open cracks by changes of measured frequencies," in Proceedings of the XVI AIMETA Congress of Theoretical and Applied Mechanics, Ferrara, Italy, Septermber 2003.

[11] A. Morassi, "Structural identification of a steel frame from dynamic test-data," Structural Engineering \& Mechanics, vol. 11, no. 3, pp. 237-258, 2001.

[12] F. Vestroni and D. Capecchi, "Damage detection in beam structures based on frequency measurements," Journal of Engineering Mechanics, vol. 126, no. 7, pp. 761-768, 2000.

[13] A. Pau, A. Greco, and F. Vestroni, "Numerical and experimental detection of concentrated damage in a parabolic arch by measured frequency variations," Journal of Vibration and Control, vol. 17, no. 4, pp. 605-614, 2010.

[14] M. Dilena and A. Morassi, "Reconstruction method for damage detection in beams based on natural frequency and antiresonant frequency measurements," Journal of Engineering Mechanics, vol. 136, no. 3, pp. 329-344, 2010.

[15] E. Giordano, N. Mendes, M. Masciotta et al., "Expeditious damage index for arched structures based on dynamic identification testing," Construction and Building Materials, vol. 265, 2020.

[16] A. Labib, D. Kennedy, and C. A. Featherston, "Crack localisation in frames using natural frequency degradations," Computers \& Structures, vol. 157, pp. 51-59, 2015.

[17] L. Rubio, J. Fernández-Sáez, and A. Morassi, "Identification of an open crack in a beam with variable profile by two resonant frequencies," Journal of Vibration and Control, vol. 24, no. 5, pp. 839-859, 2018.

[18] G. Fraraccio, A. Brügger, and R. Betti, "Experimental studies on damage detection in frame structures using vibration measurements," Shock and Vibration, vol. 17, no. 6, pp. 697-721, 2010.

[19] K. He and W. D. Zhu, "Structural damage detection using changes in natural frequencies: theory and applications," Journal of Physics: Conference Series, vol. 305, 2011.

[20] H. Y. Hwang and C. Kim, "Damage detection in structures using a few frequency response measurements," Journal of Sound and Vibration, vol. 270, no. 1-2, pp. 1-14, 2004.

[21] M. Diaferio and V. Sepe, "Modal identification of damaged frames," Structural Control and Health Monitoring, vol. 23, no. 1, pp. 82-102, 2016.

[22] M. Ge, E. M. Lui, and A. C. Khanse, "Non-proportional damage identification in steel frames," Engineering Structures, vol. 32, no. 2, pp. 523-533, 2010.

[23] R. Ditommaso, F. C. Ponzo, and G. Auletta, "Damage detection on framed structures: modal curvature evaluation using Stockwell Transform under seismic excitation," Earthquake Engineering and Engineering Vibration, vol. 14, no. 2, pp. 265-274, 2015.

[24] J. Ciambella, A. Pau, and F. Vestroni, "Modal curvature-based damage localization in weakly damaged continuous beams," Mechanical Systems and Signal Processing, vol. 121, pp. 171-182, 2019.

[25] D. Tao, Q. Ma, and S. Li, "Seismic damage detection of moment resisting frame structures using time-frequency 
features," Shock and Vibration, vol. 2018, Article ID 1086540, 2018.

[26] A. Greco and A. Pau, "Detection of a concentrated damage in a parabolic arch by measured static displacements," Structural Engineering \& Mechanics, vol. 39, no. 6, pp. 751-765, 2011.

[27] S. Caddemi and A. Greco, "The influence of instrumental errors on the static identification of damage parameters for elastic beams," Computers \& Structures, vol. 84, no. 26-27, pp. 1696-1708, 2006.

[28] J. Guo, L. Wang, and I. Takewaki, "Static damage identification in beams by minimum constitutive relation error," Inverse Problems in Science and Engineering, vol. 27, no. 10, pp. 1347-1371, 2019.

[29] M. I. Friswell, "Damage identification using inverse methods," Philosophical Transactions of the Royal Society A: Mathematical, Physical \& Engineering Sciences, vol. 365, no. 1851, pp. 393-410, 2007.

[30] P. G. Nikolakopoulos, D. E. Katsareas, and C. A. Papadopoulos, "Crack identification in frame structures," Computers \& Structures, vol. 64, no. 1-4, pp. 389-406, 1997.

[31] A. Greco and A. Pau, "Damage identification in Euler frames," Computers \& Structures, vol. 92-93, pp. 328-336, 2012.

[32] A. Greco, D. D’Urso, F. Cannizzaro, and A. Pluchino, "Damage identification on spatial Timoshenko arches by means of genetic algorithms," Mechanical Systems and Signal Processing, vol. 105, pp. 51-67, 2018.

[33] A. Yu, A. Ji, and S. Sun, "An improved multi-objective genetic algorithm and data fusion in structural damage identification," International Journal of Security and Networks, vol. 14, no. 2, pp. 95-102, 2019.

[34] A. Greco, A. Pluchino, F. Cannizzaro, S. Caddemi, and I. Caliò, "Closed-form solution based genetic algorithm software: application to multiple cracks detection on beam structures by static tests," Applied Soft Computing, vol. 64, pp. 35-48, 2018.

[35] S. R. Hoseini Vaez and N. Fallah, "Damage identification of a 2D frame structure using two-stage approach," Journal of Mechanical Science and Technology, vol. 32, no. 3, pp. 1125-1133, 2018.

[36] F. Kang, J.-J. Li, and Q. Xu, "Damage detection based on improved particle swarm optimization using vibration data," Applied Soft Computing, vol. 12, no. 8, pp. 2329-2335, 2012.

[37] P. Beena and R. Ganguli, "Structural damage detection using fuzzy cognitive maps and Hebbian learning," Applied Soft Computing, vol. 11, no. 1, pp. 1014-1020, 2011.

[38] A. J. Molina-Viedma, L. Pieczonka, K. Mendrok, E. LòpezAlba, and F. A. Diaz, "Damage identification in frame structures using high-speed digital image correlation and local modal filtration," Structural Control and Health Monitoring, vol. 27, 2020.

[39] J. Guo, J. Jiao, K. Fujita, and I. Takewaki, "Damage identification for frame structures usign vision-based measurement," Engineering Structures, vol. 199, 2019.

[40] C.-B. Yun, J.-H. Yi, and E. Y. Bahng, "Joint damage assessment of framed structures using a neural networks technique," Engineering Structures, vol. 23, no. 5, pp. 425-435, 2001.

[41] N. G. Pnevmatikos and G. D. Hatzigeorgiou, "Damage detection of framed structures subjected to earthquake excitation using discrete wavelet analysis," Bulletin of Earthquake Engineering, vol. 15, no. 1, pp. 227-248, 2017.
[42] S. Christides and D. Barr, "One-dimensional theory of cracked Bernoulli-Euler beams," International Journal of Mechanics Science, vol. 26, no. 11-12, pp. 639-648, 1984.

[43] T. G. Chondros, "The continuous crack flexibility model for crack identification," Fatigue and Fracture of Engineering Materials and Structures, vol. 24, no. 10, pp. 643-650, 2001.

[44] T. G. Chondros, A. D. Dimarogonas, and J. Yao, "A continuous cracked beam vibration theory," Journal of Sound and Vibration, vol. 215, no. 1, pp. 17-34, 1998.

[45] A. D. Dimarogonas, "Vibration of cracked structures: a state of the art review," Engineering Fracture Mechanics, vol. 55, no. 5, pp. 831-857, 1996.

[46] I. Caliò, A. Greco, and D. D'Urso, "Structural models for the evaluation of eigen-properties in damaged spatial arches: a critical appraisal," Archive of Applied Mechanics, vol. 86, pp. 1853-1867, 2016.

[47] R. Hou, J. L. Beck, X. Zhou, and Y. Xia, "Structural damage detection of space frame structures with semi-rigid connections," Engineering Structures, vol. 235, 2021.

[48] Y. Lei, Q. Li, F. Chen, and Z. Chen, "Damage identification of frame structures with joint damage under earthquake excitation," Advances in Structural Engineering, vol. 17, no. 8, pp. 1075-1087, 2014.

[49] J. N. Yang, Y. Xia, and C. H. Loh, "Damage identification of bolt connections in a steel frame," Journal of Structural Engineering, vol. 140, no. 3, 2014.

[50] C. Bilello and L. A. Bergman, "Vibration of damaged beams under a moving mass: theory and experimental validation," Journal of Sound and Vibration, vol. 274, no. 3-5, pp. 567-582, 2004.

[51] J.-H. Weng, C.-H. Loh, and J. N. Yang, "Experimental study of damage detection by data-driven subspace identification and finite-element model updating," Journal of Structural Engineering, vol. 135, no. 12, pp. 1533-1544, 2009.

[52] F. M. Mazzolani and V. Piluso, "Plastic design of seismic resistant steel frames," Earthquake Engineering \& Structural Dynamics, vol. 26, no. 2, pp. 167-191, 1997.

[53] A. Greco, F. Cannizzaro, and A. Pluchino, "Seismic collapse prediction of frame structures by means of genetic algorithms," Engineering Structures, vol. 143, pp. 152-168, 2017.

[54] A. Greco, I. Fiore, and A. Pluchino, "An optimization algorithm for the detection of damage in frame structures," in Proceedings of the 14th ECCOMAS Thematic Conference on Evolutionary and Deterministic Methods for Design, Optimization and Control (EUROGEN 2021), Athens, Greece, June 2021.

[55] CSI, CSI Analysis Reference Manual for SAP2000, Computers and Structures, Berkeley, CA, USA, 2007.

[56] U. Wilensky, "NetLogo," [Online]. Available: http://ccl. northwestern.edu/netlogo/, 1999.

[57] A. K. Chopra, "Dynamics of structures, theory and application to earthquake engineering," Prentice-Hall, Upper Saddle River, NJ, USA, 2001. 